\title{
Lesebarrieren im inklusiven Mathematikunterricht überwinden - visuelle und sprachliche Unterstützungsmaßnahmen im empirischen Vergleich
}

\author{
Anna Noll • Jürgen Roth • Markus Scholz
}

Eingegangen: 3. Januar 2019 / Angenommen: 13. Januar 2020 / Online publiziert: 30. Januar 2020

(C) Der/die Autor(en) 2020

Zusammenfassung Sprache kommt im Mathematikunterricht eine tragende Rolle zu. Schülerinnen und Schüler mit und ohne sonderpädagogischen Förderbedarf können jedoch Schwierigkeiten beim Erfassen von in Schriftsprache dargebotener Informationen haben. Das Ziel dieser Studie besteht darin, herauszufinden, wie solche Lesebarrieren reduziert werden können. Ein erleichterter Zugang zu Arbeitsaufträgen könnte durch den Einsatz von Leichter Sprache und Piktogrammen erreicht werden. Auch die Visualisierung kompletter Sätze durch Fotos könnte hilfreich sein. Zentrale Fragestellung dieser Studie ist, inwieweit die Verwendung von Leichter Sprache bzw. Leichter Sprache und Piktogrammen oder Fotos die Performanz bei der Bearbeitung mathematischer Aufgaben verbessert. Die Stichprobe bestand aus Schülerinnen und Schülern mit einem sonderpädagogischen Förderbedarf im Bereich Lernen $(N=144)$ und Lernenden ohne sonderpädagogischen Förderbedarf $(N=159)$. Die Schülerinnen und Schüler bearbeiteten Aufgaben, in welchen es um die Einführung des Bruchzahlbegriffs ging, in einer der folgenden Versionen: Leichte Sprache (EG 1), Leichte Sprache + Piktogramme (EG 2), Leichte Sprache + Fotos (EG 3) oder keine Unterstützungsmaßnahme (EG 4). Die Lesefertigkeit und der IQ der Lernenden wurde vor der Bearbeitung der Aufgaben erhoben, um vergleichbare Experimentalgruppen bilden zu können. Es zeigte sich ein signifikanter Effekt der verschiedenen Bedingungen auf die Aufgabenbearbeitung. Eine Post-Hoc-Analyse verdeutlichte, dass die Signifikanz aus dem Unterschied zwischen EG 3 und

\footnotetext{
A. Noll $(\bowtie) \cdot$ J. Roth

Institut für Mathematik, Universität Koblenz-Landau, Landau in der Pfalz, Deutschland

E-Mail: noll@uni-landau.de

J. Roth

E-Mail: roth@uni-landau.de

M. Scholz

Fakultät für Sonderpädagogik, Pädagogische Hochschule Ludwigsburg, Ludwigsburg, Deutschland E-Mail: markus.scholz@ph-ludwigsburg.de
} 
EG 4 resultierte. Die Schülerinnen und Schüler in EG 3 bearbeiteten die Aufgaben erfolgreicher als die Lernenden in EG 4.

Schlüsselwörter Leichte Sprache $\cdot$ Piktogramme $\cdot$ Fotos · Inklusion · Förderschwerpunkt Lernen

\title{
How to Overcome Reading Barriers in Inclusive Mathematics Instructions-an Empirical Comparison of Visual and Linguistic Support
}

\begin{abstract}
Language plays a significant role in mathematics education. Students with and without special educational needs may experience difficulties in capturing information presented in written language. The goal of this study is to investigate how such reading barriers can be reduced. Information can be made more accessible by linguistic simplifications like the application of easy-to-read language. Another possibility is the use of visual support, for example, symbols. Furthermore, it can be assumed that the visualization of complete sentences through photos may be helpful. This leads to the central research question: Does the use of easy-to-read language and/or enriching text with symbols or photos facilitate students' comprehension of mathematical learning tasks? The sample consisted of students with $(N=144)$ and students without special educational needs $(N=159)$. The students worked on the tasks, which dealt with the introduction of fractions, in one of the following versions: Easy-to-read language (EG 1), easy-to-read language + symbols (EG 2), easy-toread language + photos (EG 3) or neither linguistic nor visual support (EG 4). The learners' reading literacy and IQ were measured before the tasks were completed in order to form comparable experimental groups. A significant effect of the different conditions on the students' results was observed. A post hoc analysis showed that the significance resulted from the difference between EG 3 and EG 4. The students in EG 3 completed the tasks more successfully than the learners in EG 4.
\end{abstract}

Keywords Easy-to-read language $\cdot$ Symbols $\cdot$ Photos $\cdot$ Inclusion $\cdot$ Learning difficulties

\section{Einleitung}

Im Mathematikunterricht kommt Sprache eine tragende Rolle zu (Durkin und Shire 1991; Maier und Schweiger 1999; Meyer und Tiedemann 2017). Sie nimmt verschiedene Rollen ein und ist sowohl Lerngegenstand und -medium als auch Lernvoraussetzung (Meyer und Tiedemann 2017). Mit der letztgenannten Rolle von Sprache im Mathematikunterricht geht einher, dass Sprache auch eine Lernbarriere sein kann. Studien zeigen, dass Schülerinnen und Schüler mit sonderpädagogischem Förderbedarf häufig Schwierigkeiten beim Verständnis schriftsprachlich dargebotener Informationen haben (Heimlich 2016; Scholz et al. 2016a). Auch Schülerinnen und Schüler ohne sonderpädagogischen Förderbedarf erfahren Lesehürden (Prediger et al. 2015; Wilhelm 2016). Das Ziel dieser Studie besteht darin, Erkenntnisse zu 
gewinnen, wie diese Barrieren reduziert werden können. Im Sinne eines inklusiven Unterrichts sollten Lernmaterialien entwickelt werden, auf deren Inhalt möglichst alle Lernenden unabhängig von ihrer Lesefertigkeit zugreifen können. Um dieses Ziel erreichen zu können, wurden verschiedene visuelle (Piktogramme und Fotos) und sprachliche (Leichte Sprache) Unterstützungsmaßnahme im Rahmen eines quantitativ empirischen Designs verglichen. Im theoretischen Hintergrund werden zunächst verschiedene Maßnahmen zur Vermeidung von schriftsprachlichen Hürden vorgestellt sowie Erkenntnisse aus der Forschung beleuchtet. Aus der Darstellung werden Forschungsfragen abgeleitet, bevor schließlich das methodische Vorgehen dargestellt wird. Im Rahmen der anschließenden Ergebnisdarstellung werden die Forschungsfragen geklärt, wobei auch spezifisch auf interessante Extremfälle eingegangen wird. Die Ergebnisse werden im Rahmen von Abschn. 6 (Diskussion) kontextualisiert, vertieft und zu Ergebnissen anderer Studien in Beziehung gesetzt. Dabei werden auch Limitierungen der Studie beleuchtet und praktische Implikationen thematisiert.

\section{Theoretischer Hintergrund}

Ein erleichterter Zugang zu Arbeitsaufträgen kann auf unterschiedliche Weise geschaffen werden. Die vorgestellte Studie nutzt drei grundlegende Strategien:

1. die Verwendung leichter Sprache,

2. die zusätzliche Unterstützung Leichter Sprache durch Piktogramme und

3. die Kombination leichter Sprache mit fotografischen Abbildungen.

\subsection{Leichte Sprache}

Leichte Sprache wird vom Netzwerk Leichte Sprache (2006) wie folgt definiert: „Leichte Sprache ist eine sehr leicht verständliche Sprache. Man kann sie sprechen und schreiben. Leichte Sprache ist vor allem für Menschen mit Lern-Schwierigkeiten. Aber auch für andere Menschen. Zum Beispiel für Menschen, die nur wenig Deutsch können. Für Leichte Sprache gibt es feste Regeln. Menschen mit und ohne Lern-Schwierigkeiten haben die Regeln gemeinsam aufgeschrieben." Bei Leichter Sprache handelt es sich dementsprechend um ein Regelwerk zur Vereinfachung der Sprache bei schriftlichen Informationen, welches primär entwickelt wurde, um Menschen mit Lernschwierigkeiten die gesellschaftliche und politische Teilhabe zu erleichtern. Sie wurde unter maßgeblicher Mitwirkung der Zielgruppe entwickelt (Kellermann 2014), um dieser mehr Selbstbestimmung zu ermöglichen (Rüstow 2015; Zustrassen 2015). Leichte Sprache wird beispielsweise für die Vereinfachung von Manifesten und Zeitungen genutzt, wobei kritisch angemerkt werden muss, dass das Regelwerk selbst keine theoretischen Erklärungen oder empirischen Belege für die Sinnhaftigkeit der einzelnen Regeln enthält (BMAS 2014). Es bleibt unklar, warum einzelne sprachliche Merkmale als leichter $\mathrm{zu}$ verstehen erachtet werden als andere. Im Folgenden werden für die Studie wichtige Regeln exemplarisch auf Basis von Ergebnissen aus der Lesbarkeits- und Verständlichkeitsforschung kritisch betrachtet. 
Die Regeln Leichter Sprache beziehen sich auf die Wort-, Satz-, Text- und Gestaltungsebene. Nachfolgend soll nur auf die für die Studie zentralen Bereiche Wort-, Satz- und Gestaltungsebene eingegangen werden. Auf der Wortebene besagen sie, dass bekannte Wörter bevorzugt verwendet werden sollten (BMAS 2014). Diese Regel kann aus Ergebnissen der Lesbarkeits- und Verständlichkeitsforschung abgeleitet werden. Gemäß Bamberger und Vanecek (1984) ist ,ein Text umso schwieriger [...], je größer die Zahl der wenig geläufigen Wörter ist"“. Je vertrauter ein Wort ist, desto schneller und leichter kann es erkannt werden (Miller 1956). Dies lässt sich auch dadurch begründen, dass ,,besonders häufige Worte zugleich auch besonders kurze Worte sind“ (Merten 1995). Ein Text mit geringerer durchschnittlicher Wortlänge bzw. geringem Prozentsatz an Mehrsilbern (drei- und mehrsilbige Wörter) beeinflusst die Lesbarkeit positiv, da lange Wörter schwieriger mit dem Auge zu erfassen sind (Bamberger und Vanecek 1984).

Auf der Satzebene sollten laut Regelwerk kurze Sätze mit einem einfachen Satzbau verwendet werden (BMAS 2014). Auch die Nützlichkeit dieser Regel lässt sich auf Basis der Lesbarkeitsforschung belegen (Ballod 2001; Best 2006; Christmann und Groeben 1999; Langer et al. 2011). Beim Lesen ist das Erfassen von Sinneinheiten notwendig. Daher kann der Prozess des Gliederns als wesentlicher Bestandteil des Leseprozesses aufgefasst werden. Der Punkt am Ende eines Satzes stellt einen Einschnitt dar und erleichtert den Gliederungsprozess. Eine „Informationshäufung in einem langen Satz [bereitet daher] mehr Schwierigkeiten als ein kurzer Satz mit einfacher Information“ (Bamberger und Vanecek 1984). Jedoch kann die ausschließliche Verwendung kurzer und möglichst einfacher Sätze auch dazu führen, dass der Zusammenhang zwischen Sätzen unklar bleibt und vom Leser selbst erschlossen werden muss (Bamberger und Vanecek 1984; Christmann und Groeben 1999; Sutherland und Isherwood 2016; Fajardo et al. 2014). Da die Studie mit einzelnen Arbeitsaufträgen arbeitet, die in keinem gesamttextlichen Gesamtzusammenhang stehen, soll auf diese Problematik jedoch nicht weiter eingegangen werden.

Im Rahmen der Gestaltungsebene sollten laut Regelwerk alle Wörter in eine Zeile geschrieben werden, die vom Sinn her zusammengehören (BMAS 2014). Beispielsweise wurden in der vorliegenden Studie die beiden Arbeitsaufträge „Ordne die Puzzle-Teile. Beginne mit dem Größten.“ in eine Zeile geschrieben. Die erste Arbeitsanweisung kann ohne das Lesen der zweiten nicht korrekt ausgeführt werden kann. Einer sinnvollen Strukturierung und Organisation von Informationen kommt auch in der Lesbarkeitsforschung eine bedeutende Rolle zu (Langer et al. 2011; Christmann und Groeben 1999).

Zusammenfassend kann festgehalten werden, dass die Sinnhaftigkeit der großen Mehrzahl der Regeln Leichter Sprache auf Basis der Lesbarkeits- und Verständlichkeitsforschung unterstützt werden kann. Kritisch anzumerken ist, dass im Regelwerk oft eine möglichst häufige und pauschale Anwendung der Regeln empfohlen wird, obwohl dies auf Basis der Verständlichkeitsforschung nicht bestätigt werden kann (Langer et al. 2011). Eine erste empirische Untersuchung zum Vergleich der Verständniserleichterung durch Leichte Sprache mit leider nicht konkret spezifizierten anderen Vereinfachungen bei Gemeinschaftsschülerinnen und Gemeinschaftsschülern zeigt, dass „Physiktexte in Leichter Sprache weder unabhängig von den 
Personenmerkmalen noch unter deren Berücksichtigung zu einem besseren Textverständnis als die beiden anderen sprachlichen Gestaltungen zu führen scheinen“ (Kohnen et al. 2017). Die Autoren führen jedoch auch zahlreiche Einschränkungen auf, insbesondere dass sich die eingesetzten Textversionen ,von einem fachsprachlich orientierten Schulbuchtext deutlich unterschieden“ (ebd.).

\subsection{Piktogramme}

Piktogramme als Bedeutungsträger finden seit vielen Jahren Anwendung in der Unterstützten Kommunikation. Mit Unterstützter Kommunikation (UK) sind alle Kommunikationsformen gemeint, die eine fehlende Lautsprache ersetzen oder ergänzen. Technische Hilfen und Gebärden können dabei u.a. zum Einsatz kommen. Piktogramme helfen Menschen, die über keine, eine eingeschränkte oder schwer verständliche Lautsprache verfügen, sich mitteilen zu können (Abbott 2000; Detheridge und Detheridge 2002). Mittlerweile findet auch eine vermehrte Nutzung von Piktogrammen im Bereich der schriftlichen Kommunikation statt (vgl. Abbott 2000; Detheridge und Detheridge 2002).

Piktogramme oder (piktografische) Symbole sind ,einfache, schematische Bildchen, die auf einen Blick einen Begriff aktivieren oder eine Handlung auslösen“ (Ballstaedt 2012). Sie stellen in der Regel ein Wort (z. B. Kopfhörer) oder eine Einzelaussage (z. B. ,wie heißt du?“) dar. Jedes Piktogramm hat somit eine ganz bestimmte Bedeutung (vgl. Abb. 1). Piktogramme können in verschiedene Kategorien unterteilt werden, die ein Kontinuum zwischen ikonischen (abbildhaften) und symbolischen (abstrakten) Darstellungen bilden (Ballstaedt 1997, 2012). Abbott (2000), Detheridge und Detheridge (2002) sowie Poncelas und Murphy (2007) unterteilen dieses Kontinuum weiter. Letztere sprechen von transparenten, transluzenten und abstrakten Piktogramme. Transparente Piktogramme haben die höchste Ikonizität und besitzen eine offensichtliche Verbindung zum Darzustellenden, indem sie eine hohe Ähnlichkeit mit dem Objekt oder der Handlung, auf die sie sich beziehen, aufweisen. Das Piktogramm für das Wort „Kopfhörer“ ist ein Beispiel für diese Kategorie. Des Weiteren gibt es transluzente Piktogramme. Sie sind nicht unmittelbar erkennbar, aber die Verbindung zwischen dem Piktogramm und dem darzustellenden Objekt oder der darzustellenden Handlung kann leicht nachvollzogen, gelernt und erinnert werden. Sie sind häufig Teil einer Gruppe. Hat man das dahinterliegende Gestaltungskonzept verstanden, kann die Bedeutung anderer Piktogramme dieser Gruppe aus Ähnlichkeit heraus geschlussfolgert werden, wie z.B. bei ,groß“ und „klein“. Im Gegensatz zu transparenten und transluzenten Piktogrammen besitzen abstrakte Piktogramme keine Verbindung zu dem, was sie darstellen sollen. Sie sind
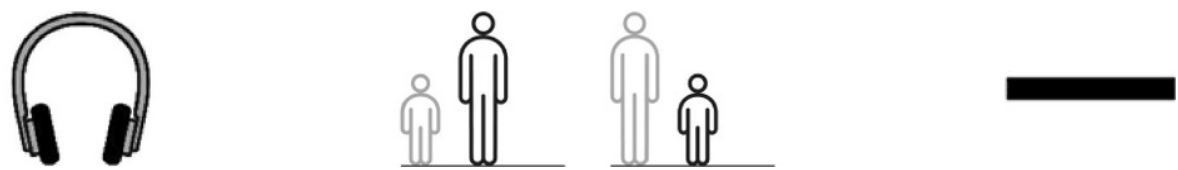

Abb. 1 Transparentes (Kopfhörer-Piktogramm aus der Metacom Symbolsammlung, Kitzinger 2015), transluzentes (groß und klein - aus der Metacom Symbolsammlung, Kitzinger 2015) und abstraktes Piktogramm (Ist-Piktogramm aus Detheridge und Detheridge 2002) 
Abb. 2 Verwendung von Schlüsselwort-Piktogrammen (Keyword Symbols)
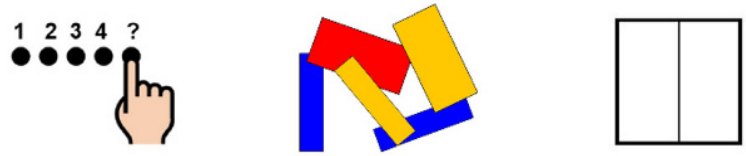

\section{Zähle die Puzzle-Teile in Quadrat A.}

vollkommen arbiträr, beispielhaft dargestellt durch ,ist“. Sie müssen ähnlich wie fremdsprachliche Vokabeln auswendig gelernt werden (vgl. Abb. 1).

Poncelas und Murphy (2007) beschreiben drei Möglichkeiten Piktogramme zur Unterstützung von Text einzusetzen. Die erste Möglichkeit besteht darin, den Satz nur mit einem Piktogramm für ein Wort zu unterstützen (Functional Symbol). Die Visualisierung aller Schlüsselwörter (Keyword Symbols) eines Satzes stellt die nächste Option dar. Dabei werden alle Schlüsselwörter, jedoch nicht die grammatischen Elemente visualisiert (vgl. Abb. 2). Die anspruchsvollste Möglichkeit ist das Symbol Reading. Hier wird jedes Wort durch ein korrespondierendes Piktogramm visualisiert.

Im deutschsprachigen Raum haben sich Scholz et al. (2016b) in den Bereichen Chemie und Mathematik sowie Zentel (2010) im Bereich der digitalen Medienbildung mit der Unterstützung von Lese- bzw. Verstehensprozessen mit Hilfe von Schlüsselwort-Piktogrammen beschäftigt. International gibt die Metaanalyse von Sutherland und Isherwood (2016) einen Einblick in den Forschungsstand. Die Ergebnisse der Studien sind zum Teil sehr unterschiedlich und auch widersprüchlich. So wird z. B. der Einsatz von Piktogrammen einerseits als positiv unterstützend angesehen (Jones et al. 2007; Scholz et al. 2016b), andererseits gibt es auch empirische Belege für eine geringe bis nicht vorhandene Erleichterung des Zugangs zu schriftlichen Informationen (Cardone 1999; Poncelas und Murphy 2007; Zentel 2010). Die Unterstützungsmaßnahme scheint dann wirksam, wenn möglichst transparente Piktogramme zum Einsatz kommen, deren Beziehung zum Realgegenstand oder der Handlung hoch bzw. deckungsgleich ist (Scholz et al. 2016b).

\subsection{Fotos}

Im Gegensatz zu Piktogrammen, die wie dargestellt in der Regel einen bestimmten Begriff oder eine bestimmte Aussage repräsentieren, können Fotos gleichzeitig mehrere der genannten Bereiche visualisieren (vgl. Abb. 3). Ihr Inhalt kann daher komplexer sein (,,complex of ideas“ Detheridge und Detheridge 2002). Während verschiedene Kategorien von Piktogrammen bezüglich ihrer Transparenz unterschieden

Abb. 3 Einsatz der Fotos Gleichzeitige Darstellung der Tabelle und der Handlung schreiben

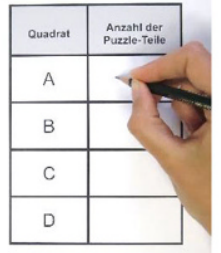

Schreibe die Zahl in die Tabelle. 
werden können, kann die Transparenz bzw. Ikonizität von Fotos generell als hoch beschrieben werden (Mirenda und Locke 1989). Es liegt in der Natur eines Fotos, dass es eine große Ähnlichkeit zu dem Objekt besitzt, das es abbildet. Diesbezüglich können daher keine verschiedenen Kategorien, die sich am bei den Piktogrammen beschriebenen Kontinuum zwischen ikonischen (abbildhaften) bzw. transparenten und symbolischen (abstrakten) Darstellungen orientieren, gebildet werden. Fotos haben in der Regel eine zusammenfassende Funktion (Detheridge und Detheridge 2002; Abbott 2000), da verschiedene Handlungen und Gegenstände gut innerhalb desselben Fotos visualisiert werden können. Sie eigenen sich daher gut dafür, den Inhalt eines ganzen Satzes oder sogar eines kompletten Absatzes zu visualisieren.

Eine von den Autoren bereits durchgeführte qualitative Studie gibt Grund zu der Annahme, dass die Visualisierung des Inhaltes kompletter Sätze eine positive Wirkung auf das Leseverständnis haben könnte (Noll et al. 2018b). In der zugrundeliegenden Studie wurden Schlüsselwort-Piktogramme zur Unterstützung des Verständnisses von Arbeitsaufträgen genutzt. Schülerinnen und Schüler wurden interviewt sowie Blickbewegungen bei der Betrachtung der Arbeitsaufträge gemessen. Es zeigte sich, dass die Teilnehmerinnen und Teilnehmer die Piktogramme eher nutzten, um den Inhalt des gesamten Satzes zu entschlüsseln, und weniger, um einzelne Begriffe oder Worte zu verstehen. Als Konsequenz daraus wurde für die vorliegende Studie die Visualisierung des kompletten Arbeitsauftrages durch ein Foto als sinnvolle Variante zur Überprüfung erachtet. Dabei soll der vollständige Satzinhalt durch jeweils ein Foto illustriert werden. Grundsätzlich würde sich der Inhalt der Satzebene auch mit Hilfe eines einzelnen oder mehrerer komplexer selbsterstellter Piktogramme darstellen lassen. Dabei hätte man aufgrund der Spezifität der geforderten Handlungen (z. B. Male die blauen Puzzle-Teile in das Quadrat.) aber nicht auf bestehende Symbolsammlungen wie Metacom (Kitzinger 2015) zurückgreifen können. Die Umsetzung wäre deshalb sehr zeitintensiv und aufwändig gewesen. Für die schulische Praxis wäre ein solches Vorgehen nicht realisierbar. Die hohe Transparenz von Fotos und somit der leicht erkennbare Bezug zum Objekt oder der realen Handlung (Mirenda und Locke 1989; Sevcik und Romski 1986) spricht neben den genannten durchführungspraktischen Aspekten ebenfalls für den Einsatz des Mediums, um Arbeitsaufträge leichter verstehen zu können.

In Bezug auf die Wirkung von Fotos auf das Leseverständnis konnten Hurtado et al. (2014) zeigen, dass die Aufnahme von Informationen bei Personen mit kognitiven Beeinträchtigungen sowohl durch Fotos als auch die Kombination von Text und Fotos unterstütz werden kann. Dabei muss in der Studie einschränkend die zusätzliche verbale Lesehilfe angemerkt werden. Trotz der hohen Nähe von Fotos zur tatsächlichen Tätigkeit oder dem tatsächlichen Objekt kann es auch bei der Verwendung von Fotos wie bei Piktogrammen zu Fehlinterpretationen kommen (Codling und Macdonald 2008; Ward und Townsley 2005).

\subsection{Mögliche Erklärungen für die Erleichterung des Leseprozesses durch die Unterstützungsmaßnahmen}

Um zu verstehen, warum die angesprochenen Maßnahmen das Verständnis erleichtern und den Leseprozess unterstützen könnten, muss man sich zunächst kurz mit 
dem Leseprozess an sich beschäftigen. Weitgehend anerkannt sind die sogenannten Zwei-Wege-Modelle des Lesens. Nach der visuellen Identifikation kann das Wort entweder direkt über einen lexikalischen Zugang abgerufen werden (orthografisches und phonologisches Lexikon) oder die einzelnen Buchstaben bzw. Silben werden phonologisch rekodiert, die Grapheme also in Phoneme umgewandelt (Dehaene 2012; Klicpera et al. 2014). Auch von fortgeschrittenen Lesern werden beide Strategien benutzt, da nicht im mentalen Lexikon vorhandene Worte auch von diesen rekodiert werden müssen. Dabei spielt das Arbeitsgedächtnis als Speicher- und Verarbeitungsinstanz für die einzelnen Buchstaben oder die Worte eines Satzes eine wichtige Rolle (Schnotz und Dutke 2004). Maßnahmen im Bereich der Leichten Sprache führen durch die Nutzung kurzer Sätze und kurzer Worte zu einer Entlastung dieses Bereichs. Die Verwendung von piktografischen Symbolen erleichtert vermutlich primär die phonologische Rekodierung unbekannter Worte, indem eine visuelle Repräsentation als zusätzlicher Hinweis bereitgestellt wird. Eine positive Wirkung von textunterstützenden Fotos könnte auf Basis des Multimedia Prinzips (Mayer 2009) angenommen werden. Es besagt, dass mit Hilfe von Wörtern und Bildern besser gelernt werden kann als mit einem der Medien alleine. Die Cognitive Theory of Mutimedia Learning sowie das Integrated Model of Text and Picture Comprehension (Schnotz 2014) liefern eine Erklärung für diese Annahme. Den beiden Theorien zufolge werden verbale und bildliche Informationen mental in zwei verschiedenen Kanälen verarbeitet. Sind sowohl Wörter als auch Bilder präsent, haben die Lernenden die Möglichkeit, verbale und visuelle mentale Modell zu konstruieren und Verbindungen zwischen diesen Modellen aufzubauen, was sich nach Mayer (2009) positiv auf den Prozess des Behaltens der Information - in unserem Fall eines Arbeitsauftrags - auswirken kann.

\subsection{Bruchzahlen}

Zunächst soll die Auswahl der Thematik (Einführung in die Bruchzahlen) begründet werden. Das Thema Bruchrechnung zeichnet sich durch seine inhaltliche Relevanz für das alltägliche Leben aus. Obwohl im Alltag oft nur wenige einfache Brüche gebraucht werden (z.B. $\frac{1}{2}, \frac{1}{4}$ und $\frac{3}{4}$ ) und auch dem Rechnen mit Brüchen eine eher geringe Bedeutung zukommt, können nur auf Basis der Bruchrechnung die lebensweltlich wichtigen Dezimalbrüche, die Dezimalschreibweise sowie das Rechnen mit Dezimalbrüchen verstanden werden (Padberg und Wartha 2017; Wartha und Wittmann 2009). Hinzu kommt, dass das Thema Bruchrechnung als Grundlage für viele weitere Bereiche der Mathematik benötigt wird (z.B.: Wahrscheinlichkeitsrechnung, Gleichungslehre) und vielfältige Zusammenhänge mit anderen Themenbereichen, wie beispielsweise der Prozentrechnung, bestehen (Padberg und Wartha 2017). Für ein Hochschulstudium (Siegler et al. 2013) sowie in der Arbeitswelt ist die Bruchrechnung weiterhin bedeutsam. Empirische Befunde, wie z.B. Analysen der mathematischen Anforderung an tätigkeitsbasiert-qualifizierten Arbeitsplätzen (Werner 2017a; Basendowski 2014), zeigen, dass für einfache Erwerbstätigkeiten mathematische Ideen bis Klasse 7, darunter die Bruchrechnung, relevant sind.

Die Wichtigkeit des Bruchzahlbegriffs und der Bruchrechnung wird auch in den Bildungsstandards der KMK für den mittleren (KMK 2003) sowie für den Haupt- 
schulabschluss (KMK 2004) im Fach Mathematik deutlich. Einen möglichen Referenzrahmen bietet des Weiteren sowohl der Rahmenlehrplan der Regelschulen (vgl. MBWJK Rheinland-Pfalz 2007) als auch der Lehrplan für Schulen mit dem Förderschwerpunkt Lernen (vgl. MBWW Rheinland-Pfalz o.J.). Im Rahmenlehrplan wird für das Thema Bruchzahlen innerhalb der Leitidee Zahl und Zahlbereiche ein Zeiteinsatz von $52 \mathrm{~h}$ empfohlen. Im Lehrplan Mathematik für Schulen mit Förderschwerpunkt Lernen ist das Thema im Feld Zahlen und Rechenoperationen eingebettet und soll auf Lernstufe sieben eingeführt werden. Auf Lernstufe acht und neun erfolgt eine Vertiefung und Wiederholung der Inhalte (ebd.).

Nachdem die Auswahl der Thematik der Bruchrechnung begründet wurde, soll nun die Grundvorstellung Teil eines Ganzen erläutert und deren Auswahl kurz begründet werden. Auf Basis der Grundvorstellung Teil eines Ganzen wird beispielsweise der Bruch $\frac{1}{4}$ wie folgt interpretiert: Ein Ganzes wird in vier gleich große Teile geteilt und eines dieser Teile wird betrachtet (Padberg und Wartha 2017). Der Nenner beschreibt also, in wie viele gleich große Teile das Ganze geteilt wird, der Zähler gibt an, wie viele dieser Teile betrachtet werden sollen. Die beschriebene Grundvorstellung gilt als vergleichsweise leicht verständlich. Dennoch wurde im Rahmen des PALMA-Projektes deutlich, dass vor der systematischen Behandlung der Bruchrechnung im Unterricht Schülerinnen und Schüler die Grundvorstellung Anteil eines Ganzen noch nicht aktivieren und noch nicht auf diese zugreifen können (Padberg und Wartha 2017; Wartha und Vom Hofe 2005). Daher wurde die Grundvorstellung Teil eines Ganzen zur Einführung des Bruchzahlbegriffs gewählt. Natürlich wäre die Einführung des Bruchzahlbegriffs auch auf Basis anderer Grundvorstellungen möglich gewesen. Um die Schülerinnen und Schüler nicht zu überfordern, schien eine Orientierung an der Grundvorstellung Teil eines Ganzen jedoch am sinnvollsten.

\section{Ziele und Forschungsfragen}

In der vorliegenden Studie ist der Vergleich der Effektivität von sprachlichen (Leichte Sprache) und visuellen (Piktogramme und Fotos) Unterstützungsmaßnahmen zentral. Die Effektivität wurde anhand der Performanz der Schülerinnen und Schüler bei der Bearbeitung mathematischer Aufgaben evaluiert. Diese Aufgaben wurden von den Lernenden entsprechend ihrer Experimentalgruppe in einer der vier Versionen bearbeitet (EG 1: Leichte Sprache, EG 2: Leichte Sprache+Piktogramme, EG 3: Leichte Sprache+Fotos, EG 4: Keine Unterstützungsmaßnahme). In den Aufgaben wird die durch das Video erfolgte die Einführung des Bruchzahlbegriffs nachgearbeitet (vgl. Abschn. 2.5). Die zentrale Forschungsfrage lautet:

Forschungsfrage 1 Verbessert die Verwendung von Leichter Sprache bzw. Leichter Sprache und Piktogrammen oder Fotos die Performanz im Sinne der Lösungsrate bei der Bearbeitung mathematischer Aufgaben, in welchen es um die Einführung der Bruchzahlen geht?

Aus dieser übergeordneten Fragestellung können die folgenden, etwas differenzierteren Forschungsfragen abgeleitet werden. 
Forschungsfrage 2 Unterscheiden sich die Schülerinnen und Schüler in den Experimentalgruppen bezüglich ihrer Performanz, wenn die Lesefertigkeit und der IQ Lernenden berücksichtigt werden?

In Forschungsfrage 2 steht der Einfluss der Lesefertigkeit und des IQs in Abhängigkeit der Zugehörigkeit der Lernenden zu den Experimentalgruppen im Fokus. Es soll beispielsweise untersucht werden, ob schwache Leserinnen und Leser von einer der Unterstützungsmaßnahmen eher profitieren als starke. Die Annahme, dass starke Leserinnen und Leser keine Unterstützungsmaßnahme benötigen, während schwache Leserinnen und Leser die Unterstützungsmaßnahmen nutzen, wird von den Ergebnissen verschiedener Studien gestützt (Jones et al. 2007; McNamara und Kintsch 1996; McNamara et al. 1996). In Zusammenhang mit Forschungsfrage 2 soll auch analysiert werden, ob Lernende unterschiedlicher Grundintelligenz von den Unterstützungsmaßnahmen gleichermaßen profitieren (vgl. Langer et al. 2011) oder ob beispielsweise Lernende, die einen niedrigen IQ-Wert erzielten, von einer der Unterstützungsmaßnahmen eher profitieren als Lernende, die einen hohen IQWert erreichten.

Forschungsfrage 3 Führen die Unterstützungsmaßnahmen zu mehr Lösungsversuchen?

In der Bearbeitung von Forschungsfrage 3 soll der Fokus von der mathematischen Richtigkeit der Lösung hin zur selbstständigen Aufgabenbearbeitung gelenkt werden. Hier interessiert nicht, ob eine Aufgabe richtig bearbeitet wurde. Zentral ist aber, ob eine Aufgabe überhaupt bearbeitet wurde. Zentral ist dementsprechend die Bearbeitung einer Aufgabe unabhängig von der inhaltlichen Richtigkeit der Lösung. Im Rahmen von Forschungsfrage 3 soll auch die Aufgabenbearbeitung von Schülerinnen und Schülern analysiert werden, die im Lesetest ein sehr niedriges Ergebnis erzielten.

Im folgenden Kapitel wird dargestellt, wie diese drei Forschungsfragen in der vorliegenden Studie untersucht wurden.

\section{Methode}

\subsection{Stichprobe}

Insgesamt nahmen 303 Schülerinnen und Schüler teil. Da das Ziel in der Gestaltung von Lernmaterial für ein inklusives Setting besteht, setzte sich die Stichprobe aus Schülerinnen und Schülern mit sonderpädagogischem Förderbedarf im Bereich Lernen (SFL, $N=144$ ) und solchen ohne sonderpädagogischen Förderbedarf (SPF, $N=159)$ zusammen. Schülerinnen und Schüler mit einem sonderpädagogischen Förderbedarf Lernen stellen mit $85 \%$ den mit Abstand größten Anteil der inklusiv beschulten Schülerinnen und Schüler mit SPF in Rheinland-Pfalz dar. Die Schülerinnen und Schüler verteilten sich auf 24 Schulklassen an zwölf Schulen, darunter 
neun Förderschulen mit dem Förderschwerpunkt Lernen, zwei Schwerpunktschulen ${ }^{1}$ (Integrierte Gesamtschulen) und eine Realschule plus ${ }^{2}$ in Rheinland-Pfalz. Dementsprechend waren Schülerinnen und Schüler mit SFL, die zum Erhebungszeitpunkt inklusiv beschult wurden $(N=9)$, und Schülerinnen und Schüler mit SFL, die an Förderschulen mit dem Schwerpunkt Lernen beschult wurden $(N=135)$, in der Stichprobe vertreten. Die Stichprobe kann daher als geschichtete Gelegenheitsstichprobe angesehen werden. Inhaltliche Voraussetzung für die Teilnahme an der Studie war, dass das Thema rationale Zahlen im bisherigen Unterricht der Sekundarstufe I bei den teilnehmenden Schülerinnen und Schüler noch nicht behandelt wurde. Der Großteil der Schülerinnen und Schüler $(N=295)$ befand sich zum Erhebungszeitpunkt im fünften, sechsten oder siebten Schulbesuchsjahr (MAlter $=11,12 ;$ SDAlter $=1,044 ;$ MSchulbesuchsjahr= 5,52; SDSchulbesuchsjahr=0,833). An der Studie nahmen jeweils komplette Schulklassen teil, wobei die Schülerinnen und Schüler innerhalb der Klassen anhand ihrer kognitiven Fähigkeit, ihrer Lesefertigkeit und ihrer Klassenzugehörigkeit auf die vier Experimentalgruppen parallelisiert wurden (vgl. Abschn. 4.4).

\subsection{Aufgaben}

Die genutzten Aufgaben (Verfügbar unter: http://dms.uni-landau.de/noll/ lernmaterial/) sind so konzipiert, dass die Schülerinnen und Schüler sie in Einzelarbeit in ihrem individuellen Tempo bearbeiten können. Um den Lernenden die selbstständige Bearbeitung zu erleichtern, wird in kleinen Schritten vorgegangen

Abb. 4 Puzzleteile

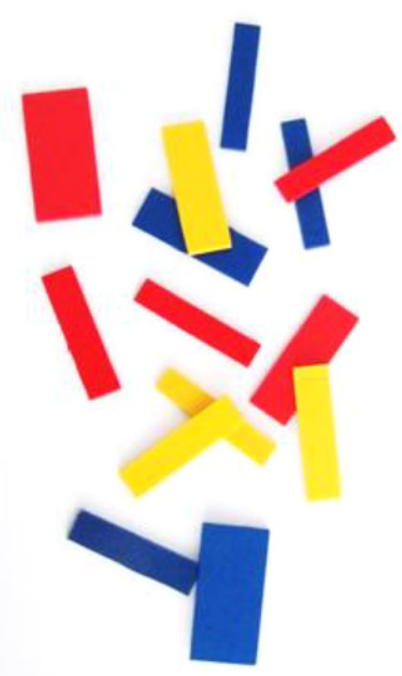

\footnotetext{
1 In Schwerpunktschulen werden Schülerinnen und Schüler mit und ohne SPF gemeinsam unterrichtet (vgl. Laubenstein et al. 2015, S. 13 ff.).

2 Die Schulform Realschule plus ist aus der Zusammenführung von Haupt- und Realschulen entstanden.
} 


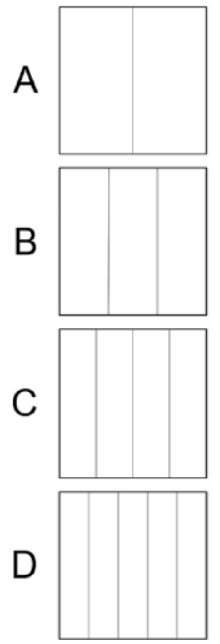

(Scherer 1999; Werner 2017b). Gegenständliche Materialien in Form von Puzzleteilen (Abb. 4) und einer Schablone (Abb. 5) kommen dabei unterstützend zum Einsatz. Sie stehen allen SuS für die Aufgabenbearbeitung zur Verfügung. Alle Bruchzahlen in den Aufgaben sind mit den Puzzleteilen und der Schablone darstellbar (Halbe, Drittel, Viertel und Fünftel) und die Grundvorstellung Teil eines Ganzen ist für die Aufgabenbearbeitung zentral (vgl. Abschn. 2.5). Die Aufgaben setzen sich aus zwei Komplexen zusammen. Im ersten Teil geht es um das Vertraut machen mit dem Material und der jeweiligen Unterstützungsmaßnahme. Zunächst legen die Schülerinnen und Schüler die verschieden großen Puzzleteile (Abb. 4) in die Quadrate A, B, C und D auf der Schablone (Abb. 5). Dann zählen die Lernenden die Puzzleteile und notierten ihre Ergebnisse in einer Tabelle. Hier werden folglich keine für die Schülerinnen und Schüler neuen mathematischen Inhalte behandelt.

Nach der Bearbeitung des ersten Aufgabenkomplexes erhalten die Lernenden einen Input über Bruchzahlen in Form eines Stop-Motion-Videos (Verfügbar unter: http://www.dms.uni-landau.de/noll/lernmaterial/). Die Lernenden starten dieses Video selbstständig auf dem ihnen zur Verfügung stehenden Laptop (mit Kopfhörern), sobald sie mit der Bearbeitung des ersten Aufgabenteils fertig sind. Eine Kontrolle des ersten Aufgabenteils oder ein Gespräch über das Lernvideo findet nicht statt. Im Video werden zählend zunächst der Nenner und der Zähler bestimmt. Diese Zählergebnisse werden unter- und oberhalb eines gegebenen Bruchstrichs notiert, woraus sich ein Darstellungswechsel ergibt. Die Idee des Anteils, in welcher der Bruch als eine Zahl aufgefasst wird, bleibt dabei eher implizit. Im Lernvideo ist die Grundvorstellung Anteil eines Ganzen (vgl. Abschn. 2.5) zentral. Sie wird (vgl. Abb. 6 und 7) in Anlehnung an Padberg und Wartha (2017) zunächst von einer Sprecherin anhand des Stammbruches $\frac{1}{4}$ erklärt: ,Wir haben schon entdeckt, dass Quadrat C aus vier gleich großen Teilen besteht. Nur einer dieser Teile ist blau. Das bedeutet, dass die blaue Fläche ein Viertel des ganzen Quadrates bedeckt“. Anschließend wird sie für 
Abb. 6 Ausschnitt 1 aus dem Lernvideo
Anzahl blau : $\mathbb{1}$

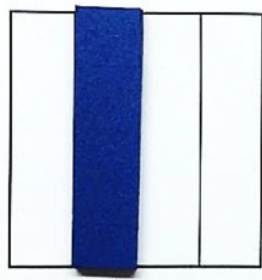

$\frac{1}{4}$
Abb. 7 Ausschnitt 2 aus dem Lernvideo

\section{Anzahl gelb: 2}

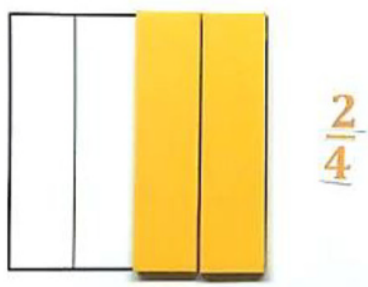

Anzahl: 4

den Bruch $\frac{2}{4}$ wiederholt. Parallel zur mündlichen Erklärung wird im Video das zugehörige Zahlzeichen eingeführt (vgl. Padberg und Wartha 2017).

Im zweiten Aufgabenkomplex wenden die Schülerinnen und Schüler den Inhalt des Lernvideos an. Dies geschieht zunächst, indem sie zwei Grundaufgaben ${ }^{3}$ bearbeiten, die für die Einführung des Bruchzahlbegriffs zentral sind (Padberg und Wartha 2017). In den Grundaufgaben sind jeweils zwei der drei Komponenten ( $A n-$ teil, Teil, Ganzes) gegeben, die dritte soll von den Schülerinnen und Schülern ergänzt werden (vgl. Abb. 8 und 9).

Weitere Aufgaben des zweiten Aufgabenkomplexes, die aus Platzgründen hier nicht abgedruckt sind, beschäftigen sich mit dem Vergleichen und Ordnen von Bruchzahlen nach ihrer Größe. Dabei wird kein losgelöster symbolischer Vergleich durch die Bildung eines gemeinsamen Hauptnenners angestrebt. Stattdessen sollen

\footnotetext{
3 Als Grundaufgaben bezeichnen Padberg und Wartha (2017) Aufgaben bei denen das Ganze, der Anteil oder der Teil gesucht ist. Die beiden übrigen Größen sind dabei gegeben.
} 
Abb. 8 Aufgabe 2.a

Abb. 9 Aufgabe 2.b
Schreibe die Bruchzahl auf.

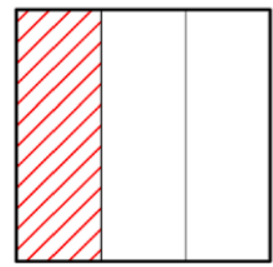

Male die passende Fläche an.

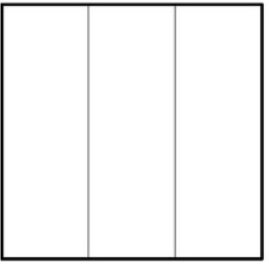

die Lernenden die Grundvorstellung Teil eines Ganzen aktivieren, selbst erstellte Abbildungen nutzen und die größere Bruchzahl auf ikonischer Ebene bestimmen (vgl. Wartha und Wittmann 2009; Padberg und Wartha 2017). Es wird daher, wie in der einschlägigen Literatur empfohlen, auf einer anschaulichen Ebene gearbeitet (Padberg und Wartha 2017; Scherer 1999). Am Ende sollen die Schülerinnen und Schüler selbst kreativ werden, indem sie sich eine beliebige Bruchzahl ausdenken, diese benennen und zeichnerisch darstellen.

Im Folgenden wird die Realisierung der vier Untersuchungsbedingungen (Keine Unterstützungsmaßnahme, Leichte Sprache, Piktogramme, Fotos) erläutert. Die nicht vereinfachten Arbeitsaufträge (Verfügbar unter: http://dms.uni-landau.de/noll/ lernmaterial/) wurden von Experten (Mathematikdidaktikerinnen und Mathematikdidaktiker, die an Schule und/oder Hochschule tätig sind) verfasst. Die von den Lernenden gewünschten Handlungen wurden dargestellt, die Experten formulierten die dazu passenden Arbeitsaufträge. Den Experten war bekannt, dass es sich um Arbeitsaufträge für Schülerinnen und Schüler mit und ohne SPF, vorwiegend in Klassenstufe 5, handelt. Basierend auf Überschneidungen in den von den Experten versprachlichten Arbeitsaufträgen wurden die Arbeitsaufträge für die Bedingung keine Unterstützungsmaßnahme formuliert. Zur Überprüfung eines angemessenen sprachlichen Anspruchs der Arbeitsaufträge wurde der Lesbarkeitsindex (LIX) nach Björnsson berechnet. In die Berechnung des LIX geht die Satzlänge (Gesamtzahl der Wörter dividiert durch die Anzahl der Sätze) sowie der Prozentsatz langer Wörter (Anzahl der langen Wörter dividiert durch die Gesamtzahl der Wörter mal 100) ein. Als Tool zur Berechnung wurde die Download-Version auf der Internetseite Psychometrica (http://www.psychometrica.de/lix.html) genutzt. Die mit dem LIX-Verfahren errechnete Textschwierigkeit der Arbeitsaufträge lag bei 43 (niedrige Textschwierigkeit) mit einer durchschnittlichen Satzlänge von 12 Wörtern. 
Die Umsetzung der Unterstützungsmaßnahme Leichte Sprache (Verfügbar unter: http://dms.uni-landau.de/noll/lernmaterial/) soll anhand zweier Beispiele illustriert werden. Gemäß des Regelwerks Leichte Sprache sollen bekannte und kurze Wörter bevorzugt benutzt werden (BMAS 2014). Dementsprechend wurde beispielsweise „schreiben“ statt „,notieren“ und „malen“ statt „schraffieren“ verwendet. Kritisch angemerkt werden muss, dass im Regelwerk offenbleibt, was genau unter einem bekannten Wort verstanden werden kann. In den vorliegenden Aufgaben wurden bekannte Wörter u.a. mit Hilfe der Häufigkeitswortliste von Bamberger und Vanecek (1984) definiert. Des Weiteren wird postuliert, dass kurze und einfache Sätze verwendet werden sollen, die nur eine Aussage enthalten (BMAS 2014). Die Arbeitsanweisung ,Schreibe die Bruchzahlen nach ihrer Größe geordnet auf.“ wurde dementsprechend durch zwei Sätze ausgedrückt: „Schreibe die Bruchzahlen auf. Beginne mit der Größten." Dass der sprachliche Anspruch der Arbeitsaufträge für das Alter der teilnehmenden Schülerinnen und Schüler angemessen ist, wurde ebenfalls mit Hilfe des LIX-Verfahrens überprüft. Die errechnete Textschwierigkeit der Arbeitsaufträge lag bei 35 (sehr niedrige Textschwierigkeit) mit einer durchschnittlichen Satzlänge von 5 Wörtern.

Tab. 1 Exemplarischer Vergleich der vier Bedingungen

\begin{tabular}{ll}
\hline Bedingung & Arbeitsauftrag \\
\hline Leichte Sprache & Male die passende Fläche an
\end{tabular}

Leichte Sprache+

Piktogramme

Leichte Sprache + Fotos

Keine Unterstützungsmaßnahme

\section{Male die passende Fläche an.}

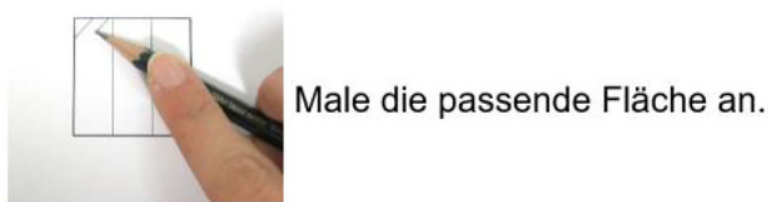

Schraffiere den Anteil des Quadrats, der durch den Bruch angegeben wird

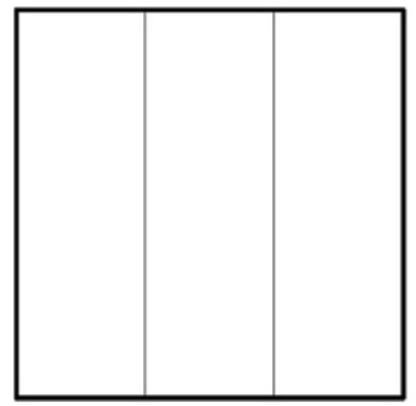


Es kann festgehalten werden, dass sowohl die Verständlichkeit der Arbeitsaufträge in Leichter Sprache (LIX=35) als auch die nicht vereinfachten Arbeitsaufträge $(\mathrm{LIX}=43)$ für das Alter der teilnehmenden Schülerinnen und Schüler geeignet zu sein scheinen. Die Textschwierigkeit der Arbeitsaufträge in Leichter Sprache kann im Sinne des LIX-Verfahrens als sehr leicht gemessen am Alter der Lernenden beschrieben werden. Die nicht vereinfachten Arbeitsaufträge sind für das Alter der Schülerinnen und Schüler angemessen.

Bei der Gestaltung der Piktogramme und der Fotos waren die folgenden Kriterien zentral (vgl. Tab. 1):

1. Möglichst hohe Transparenz

2. Sinnvolle inhaltliche Ausrichtung

3. Übersichtliche Darstellung im Arbeitsheft

Sowohl bei der Gestaltung der Piktogramme als auch bei der Entwicklung der Fotos wurde auf eine möglichst hohe Transparenz geachtet. Im Rahmen der Piktogramme wurde dies durch die ausschließliche Verwendung von transluzenten und transparenten Piktogrammen angestrebt. Im Rahmen der Fotos sollte eine möglichst hohe Transparenz durch die Einbindung der konkreten Lernumgebung erreicht werden. Die inhaltliche Ausrichtung der beiden Unterstützungsmaßnahmen kann folgendermaßen zusammengefasst werden: Während die Piktogramme auf Wortebene den Inhalt der Schlüsselwörter eines Satzes illustrieren, sind die Fotos auf der Satzebene anzusiedeln. Sie sollen die Gesamthandlung des Arbeitsauftrages, also den Satzinhalt, darstellen. In manchen Aufgaben war es nicht möglich, dass alle Informationen des Satzes in den Fotos dargestellt werden. In diesen Fällen wurden die Fotos im Sinne eines methodischen Hinweises verwendet. Um eine übersichtliche Darstellung im Arbeitsheft zu erreichen, wurde u. a. auf eine große räumliche Nähe der zueinander in Bezug stehenden sprachlichen und visuellen Entitäten geachtet. Im Fall der Piktogramme bedeutet dies, dass sich die Piktogramme unmittelbar über den Schlüsselwörtern befinden, auf die sie sich beziehen. Die Fotos befinden sich neben den Arbeitsaufträgen, die sie darstellten. Tab. 1 stellt die vier Bedingungen exemplarisch anhand einer Aufgabe gegenüber.

\subsection{Kodierung der Aufgabenbearbeitung}

Da die Aufgaben als Erhebungsinstrument dienen, soll im Folgenden kurz auf deren Kodierung eingegangen werden. Die Schülerantworten bei der Bearbeitung der Aufgaben wurden basierend auf der mathematischen Korrektheit der Lösung als richtig (1) oder falsch (0) kodiert. Insgesamt besteht der erste Aufgabenkomplex aus vier Items. Der zweite Aufgabenkomplex setzt sich aus 23 Items zusammen. Daraus ergibt sich ein maximaler Summenscore von 4 bzw. 23.

Mit Blick auf Forschungsfrage 3 ( Führen die Unterstützungsmaßnahmen zu mehr Lösungsversuchen?) wurde eine weitere, verfeinerte Kodierung vorgenommen (vgl. Abschn. 5.3). Dazu wurde die Kategorie falsch aufgespalten in ausgelassen (0) und falsch bearbeitet (1). Eine mathematisch korrekte Lösung wurde mit (2) kodiert. Da genug Zeit zur Verfügung stand, um sämtliche Aufgaben zu bearbeiten, wurden alle nicht bearbeiteten Items als ausgelassen gewertet und mit (0) kodiert. Als bearbeitet 
wurde eine Aufgabe gewertet, wenn ein Ergebnis notiert wurde, welches einen Bezug zur Aufgabe hatte, aber nicht korrekt war (z. B. falsche Zahlenwerte). Ein Ergebnis ohne inhaltlichen Bezug zur Aufgabe (z.B. ein beliebig gemaltes Bild) wurde als ausgelassen kodiert.

Die Kodierung des Lernmaterials wurde zunächst von verschiedenen Personen, denen eine Musterlösung zur Verfügung stand, vorgenommen. Anschließend wurde die vorgenommene Auswertung von der Erstautorin überprüft. Bei nicht eindeutigen Schülerlösungen wurde ein Konsens auf Basis einer kommunikativen Validierung (Bryman 2012) gefunden. So sollte der Einfluss von Flüchtigkeitsfehlern im Rahmen der zur Verfügung stehenden Kapazitäten auf ein Minimum reduziert und die Auswertungsobjektivität sichergestellt werden (Bühner 2006).

\subsection{Design}

Aufgrund der in der Stichprobe vorherrschenden Heterogenität wurde eine Randomisierung der Schülerinnen und Schüler als nicht ausreichend für die Bildung vergleichbarer Experimentalgruppen erachtet. Daher wurde zunächst die kognitive Fähigkeit der Schülerinnen und Schüler mit dem CFT 20-R (Weiß und Weiß 2006) sowie deren basale Lesefertigkeit mit dem SLS 2-9 (Wimmer und Mayringer 2014) erhoben. Anhand dieser Kontrollvariablen sowie ihrer Klassenzugehörigkeit wurden die Schülerinnen und Schüler in vier Experimentalgruppen parallelisiert.

Die Schülerinnen und Schüler bearbeiteten die Aufgaben in einer der in Tab. 2 dargestellten Bedingungen. Es gab bei der Bearbeitung keine zeitlichen Vorgaben. Die Testzeit wurde adaptiv an jede Klasse angepasst. Die durchschnittliche Bearbeitungszeit über alle Experimentalgruppen hinweg betrug $21 \mathrm{~min}$ und $11 \mathrm{~s}$ mit einem Range von 9-38 min. Die durchschnittliche Bearbeitungszeit unterschied sich nur minimal zwischen den Experimentalgruppen (EG 1: 21,00; EG 2: 21,44; EG 3: 21,15; EG 4: 20,47).

\subsection{Instrumente}

Der CFT 20-R ist ein ,,sprachfreier Intelligenztest mit figuralen, anschaulichen Denkaufgaben, [der] wesentliche Teile des allgemeinen intellektuellen Niveaus (Grundintelligenz)“ (Weiß und Weiß 2006) erfasst. Diese Grundintelligenz kann gemäß der Cattell-Horn-Intelligenztheorie beschrieben werden als „Fähigkeit, neue und unvertraute Probleme durch gezieltes schlussfolgerndes, logisches Denken zu lösen und nicht nur durch automatisierten Abruf von erworbenem Wissen, erlernten Gewohnheiten oder Schemata zu bewältigen“ (Renner und Michley 2015). Da das Thema

Tab. 2 Experimentalgruppen

\begin{tabular}{lll}
\hline Experimentalgruppe & Bedingung & Anzahl der Schülerinnen und Schüler \\
\hline EG 1 & Leichte Sprache & 77 \\
EG 2 & Leichte Sprache + Piktogramme & 75 \\
EG 3 & Leichte Sprache + Fotos & 74 \\
EG 4 & Keine Unterstützungsmaßnahme & 77 \\
\hline
\end{tabular}


rationale Zahlen den Schülerinnen und Schüler weitestgehend unbekannt ist, wird genau diese Fähigkeit bei der Bearbeitung der Aufgaben gefordert.

Der SLS 2-9 wird im Manual als „ökonomisches Verfahren zur Erfassung von Unterschieden in [der] basale[n] Lesefertigkeit“" (Wimmer und Mayringer 2014) beschrieben. Basale Lesefertigkeiten meint das möglichst schnelle und mühelose Lesen einzelner Sätze. Die Grundidee des SLS 2-9 besteht in der möglichst raschen Beurteilung von inhaltlich relativ einfachen Sätzen nach ihrer Richtigkeit (Wimmer und Mayringer 2014). Um auf die in Abschn. 4.2 vorgestellten Aufgaben zugreifen zu können, ist es nicht nötig, den Zusammenhang eines komplexen schriftsprachlich dargebotenen Textes zu erfassen. Vielmehr ist das Erfassen einzelner Sätze zentral. Dementsprechend kann das Konstrukt der basalen Lesefertigkeit, welches im SLS 2-9 erfasst wird, als geeignetes Maß für die Bildung vergleichbarer Experimentalgruppen beschrieben werden. Da die Bearbeitungszeit für den SLS 2-9 auf 3 min beschränkt ist, spielt neben der basalen Lesefertigkeit auch die Leseflüssigkeit eine Rolle (vgl. Rosebrock und Nix 2006). Hinzu kommt, dass die Anzahl der in drei Minuten richtig beurteilten Sätze hoch mit der globalen Lesefertigkeit, welche auch komplexere Anforderungen beinhaltet, korreliert $(\mathrm{r}=0,64)$ (ebd.). Dementsprechend wird auch die sehr aufwendig zu erhebende globale Lesefertigkeit, obwohl sie für die Bearbeitung der entwickelten Aufgaben nicht zentral zu sein scheint, im SLS 2-9 berücksichtigt.

\section{Ergebnisse}

In den folgenden Abschnitten soll das Textverstehen der Schülerinnen und Schüler anhand ihrer Performanz bei der Aufgabenbearbeitung analysiert werden. Diese Analyse der Ergebnisse geschieht sowohl in Abhängigkeit des mathematischen Inhalts, der Einführung der Bruchzahlen (Abschn. 5.1), als auch generell, d. h. losgelöst vom mathematischen Inhalt der Aufgaben (Abschn. 3.5).

\subsection{Experimentalgruppenvergleich}

Die aus der Aufgabenbearbeitung resultierenden Daten wurden auf ihre Eignung für die Durchführung einer einfaktoriellen Varianzanalyse geprüft. Es zeigte sich, dass diese erfüllt waren (Eid et al. 2010; Field 2013; Rasch et al. 2010). Eine ANOVA mit dem Summenscore als abhängiger Variable und der Bedingung als Faktor (Zugehörigkeit zu EG 1 Leichte Sprache, EG 2 Leichte Sprache + Piktogramme, EG 3 Leichte Sprache + Fotos oder EG 4 Keine Unterstützungsmaßnahme) führt zu einem signifikanten Ergebnis $(F(3,30)=2,88 ; p=0,036)$. Eine Post-Hoc-Analyse ${ }^{4}$ zeigt, dass die Signifikanz aus der Differenz zwischen EG 3 (Leichte Sprache + Fotos) und EG 4 (Keine Unterstützungsmaßnahme) resultiert $(p=0,041)$.

Ein Vergleich der Mittelwerte macht deutlich (vgl. Abb. 10 und Tab. 3), dass die Schülerinnen und Schüler in EG 3 (Leichte Sprache+Fotos) einen signifikant

\footnotetext{
4 Als Post-Hoc-Analyse wurde ein Gabriel-Test gewählt. Dieser verfügt über eine große Power sowie eine hohe Kontrolle des Fehlers 1. Art (Field 2013, S. 459).
} 
Abb. 10 Summenscore Mittelwerte

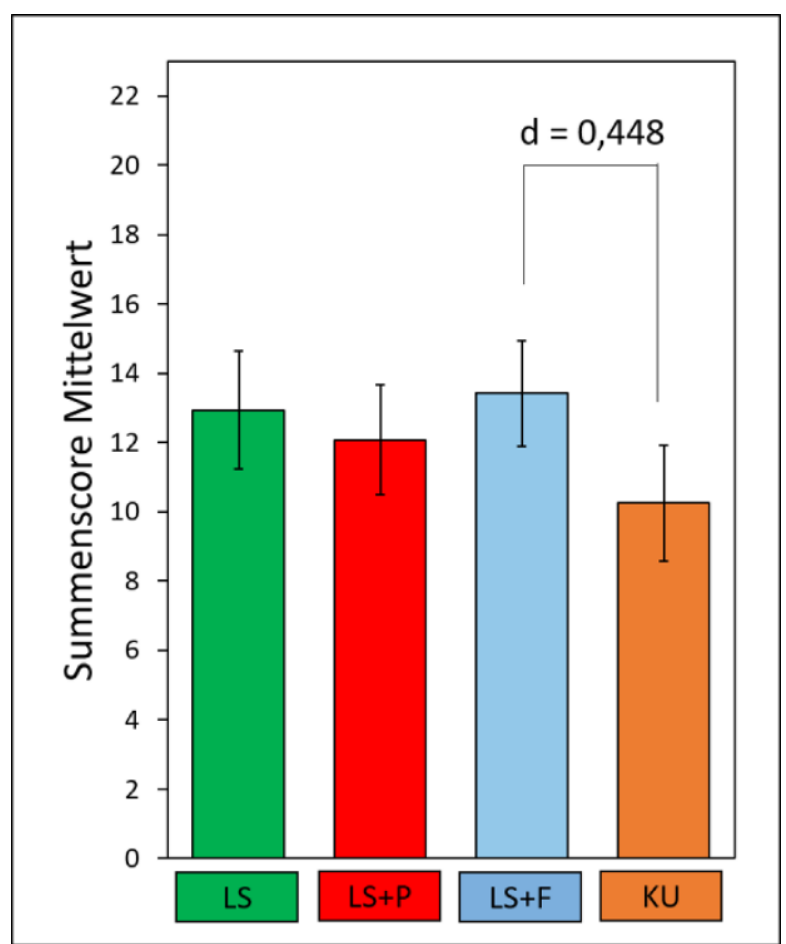

höheren Summenscore bei der Bearbeitung der Aufgaben erreichen als die Lernenden in Experimentalgruppe 4 (Keine Unterstützungsmaßnahme). Es handelt sich mit $d=0,448^{5}$ nach Cohen um einen kleinen bis mittleren Effekt. Des Weiteren zeigt der Vergleich der Mittelwerte, dass die Schülerinnen und Schüler in EG 2 (Leichte Sprache+Piktogramme) im Durchschnitt einen etwas schlechteren Summenscore erzielen als die Lernenden in EG 1 (Leichte Sprache). Dieser Unterschied ist relativ gering und nicht signifikant.

Tab. 3 Deskriptive Daten zum Summencore

\begin{tabular}{lllllll}
\hline & $N$ & M & SD & KI & Min & Max \\
\hline LS & 77 & 12,94 & 7,03 & {$[11,34 ; 14,53]$} & 0 & 23 \\
LS + P & 75 & 12,08 & 7,53 & {$[10,35 ; 13,81]$} & 0 & 23 \\
LS + F & 74 & 13,42 & 6,66 & {$[11,88 ; 14,96]$} & 0 & 23 \\
KU & 77 & 10,25 & 7,46 & {$[8,55 ; 11,94]$} & 0 & 23 \\
Gesamt & 303 & 12,16 & 7,26 & {$[11,34 ; 12,98]$} & 0 & 23 \\
\hline
\end{tabular}

$N$ Anzahl der Probanden

Die folgenden Anmerkungen beziehen sich auf den Summenscore: $M$ Mittelwert, SD Standardabweichung, KI 95\% Konfidenzintervall, Min Minimum, Max Maximum, LS Leichte Sprache, $L S+P$ Leichte Sprache + Piktogramme, $L S+F$ Leichte Sprache + Fotos, $K U$ Keine Unterstützungsmaßnahme

\footnotetext{
5 Nach Cohen wird ein Effekt bis $d=0,2$ als klein bezeichnet, ab $d=0,5$ spricht man von einem mittleren Effekt und ab $d=0,8$ gilt ein Effekt als stark (Bühner 2006, S. 121).
} 


\subsection{Einfluss der Prädiktoren}

Im diesem Abschnitt wird der Einfluss der Lesefertigkeit und des IQs auf die Bearbeitung der Aufgaben betrachtet. Es wird analysiert, ob sich die Wirkung dieser Variablen zwischen den Experimentalgruppen unterscheidet. Um herauszufinden, ob es einen Unterschied bezüglich der im vorherigen Abschnitt dargestellten Ergebnisses unter Berücksichtigung der Zugehörigkeit der Lernenden zu den vier Experimentalgruppen gibt, wird zunächst die Interaktion zwischen der basalen Lesefertigkeit (SLS 2-9) und der Bedingung betrachtet. Dazu wird die Annahme der Homogenität der Regressionssteigungen überprüft (Field 2013). Die Haupteffekte der vier verschiedenen Bedingungen sowie der basalen Lesefertigkeit werden dabei berücksichtigt (ebd.).

Die Annahme der Homogenität der Regressionssteigungen besagt, dass die Beziehung zwischen der Kovariaten Lesefertigkeit und der abhängigen Variablen Summenscore ähnlich für die verschiedenen Stufen der unabhängigen Variable (Zugehörigkeit zu EG 1 Leichte Sprache, EG 2 Leichte Sprache + Piktogramme, EG 3 Leichte Sprache + Fotos oder EG 4 Keine Unterstützungsmaßnahme) ist (Field 2013). Da die Interaktion zwischen Bedingung und Lesefertigkeit mit $p=0,968$ nicht signifikant ist, wird die Annahme der Homogenität der Regressionssteigungen nicht verletzt und es kann darauf geschlossen werden, dass die Beziehung zwischen Lesefertigkeit und Summenscore in jeder Experimentalgruppe ähnlich ist. Der folgende Scatterplot (Abb. 11) visualisiert dieses Ergebnis. Abb. 11 zeigt, dass die Beziehung zwischen Lesefertigkeit und Summenscore in allen Experimentalgruppen ähnlich ist: Mit steigender Lesefertigkeit steigt der Summenscore, wobei die Schülerinnen und Schüler in EG 3 (Leichte Sprache+Fotos) durchschnittlich den höchsten und die Schülerinnen und Schüler in EG 4 (Keine Unterstützungsmaßnahme) durchschnittlich den niedrigsten Summenscore erreichen. Die Steigung der vier Linien, welche EG 1 (Leichte Sprache), EG 2 (Leichte Sprache+Piktogramme), EG 3 (Leichte Sprache + Fotos) und EG 4 (keine Unterstützungsmaßnahme) darstellen, ist sehr ähnlich und der Abstand zwischen den verschiedenen Bedingungen sowie deren Reihenfolge kann als konstant beschrieben werden. Die Bedeutung dieser Konstanz soll an einem konkreten Bespiel veranschaulicht werden. Betrachtet man die Schülerinnen und Schüler mit niedriger Lesefertigkeit, beispielsweise mit einem Lesequotienten ${ }^{6}$ von 75 (unterdurchschnittliche Lesefertigkeit), im Vergleich zu Lernenden mit hoher Lesefertigkeit, beispielsweise mit einem Lesequotienten von 125 (überdurchschnittliche Lesefertigkeit), so ist der Effekt der Bedingung äquivalent: Die Schülerinnen und Schüler in EG 4 (Keine Unterstützungsmaßnahme) erreichen im Durchschnitt den niedrigsten Summenscore, die Lernenden in EG 3 (Leichte Sprache+Fotos) erreichen im Durchschnitt den höchsten Summenscore. Bei starken und schwachen Lesern ist der Effekt der Bedingung dementsprechend vergleichbar. Obwohl sich die vier Linien teilweise schneiden, ist ihre Steigung sehr ähnlich und führt, wie bereits erwähnt, mit $p=0,968$ zu keiner signifikanten Interaktion zwischen Bedingung und Lesefertigkeit.

\footnotetext{
${ }^{6}$ Die Anzahl der gelesenen und korrekt beantworteten Sätze wurde in einen „Lesequotienten“, der wie der Intelligenzquotient skaliert ist (Mittelwert 100; Standardabweichung 15), umgerechnet.
} 


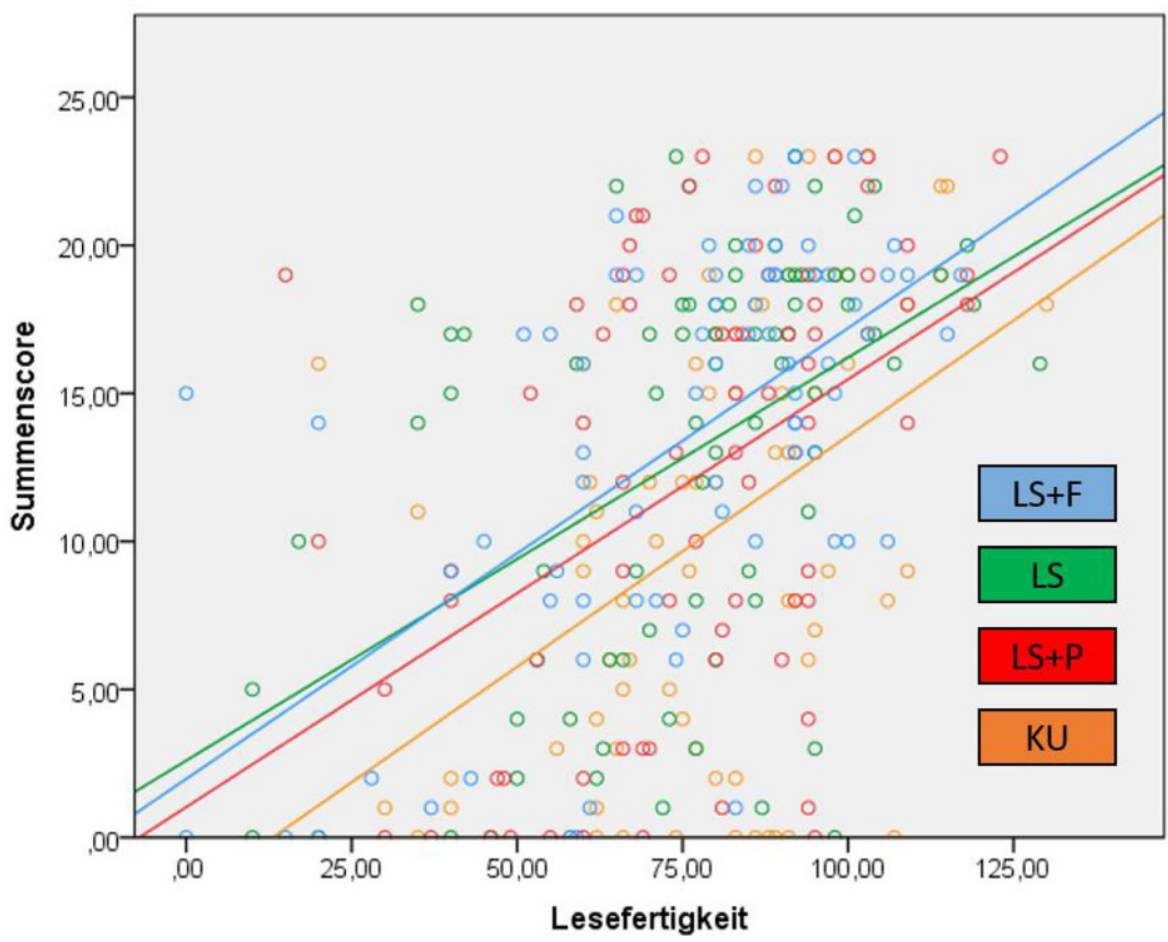

Abb. 11 Scatterplot Lesefertigkeit und Summenscore $(L S+F$ Leichte Sprache + Fotos, $L S$ Leichte Sprache, $L S+P$ Leichte Sprache + Piktogramme, $K U$ Keine Unterstützungsmaßnahme)

Um zu analysieren, ob sich die Wirkung der Unterstützungsmaßnahmen in Abhängigkeit von dem IQ-Wert der Schülerinnen und Schüler ändert, wird ähnlich vorgegangen. Die Interaktion zwischen Bedingung und IQ führt mit $p=0,613$ zu einem nicht signifikanten Ergebnis. Dementsprechend wird auch bezüglich des IQs die Annahme der Homogenität der Regressionssteigungen nicht verletzt, woraus folgt, dass die Beziehung zwischen IQ und Summenscore in jeder Experimentalgruppe ähnlich ist.

\subsection{Lösungsversuche}

Nachdem der Unterschied zwischen den Experimentalgruppen hinsichtlich der Anzahl der mathematisch korrekt bearbeiteten Items analysiert wurde, soll im Folgenden der Zugriff auf die Aufgaben unabhängig von der Richtigkeit des Ergebnisses untersucht werden.

Die aus der beschriebenen Kodierung resultierenden Häufigkeiten der Kategorien ausgelassen und falsch erfüllen nicht alle für die Durchführung eines metrischen Verfahrens notwendigen Voraussetzungen (Eid et al. 2010; Field 2013; Rasch et al. 2010). Es liegt keine Normalverteilung vor und die Varianzen in den Gruppen unterscheiden sich signifikant. Dementsprechend wurde die non-parametrische Alter- 
native zur einfaktoriellen ANOVA, der Kruskal-Wallis-Test, eingesetzt (Rasch et al. 2010).

Zunächst soll die Anzahl der ausgelassenen Items betrachtet werden. Diese unterscheidet sich signifikant zwischen den Bedingungen $(H(3)=15,77 ; p=0,001)$. Abb. 12 zeigt, dass EG 1 (Leichte Sprache) bezüglich der Anzahl der ausgelassenen Items einen Median von 1, EG 2 (Leichte Sprache+Piktogramme) einen Median von 3, EG 3 (Leichte Sprache+Fotos) einen Median von 0 und EG 4 (Keine Unterstützungsmaßnahme) einen Median von 4 erreicht. Ein paarweiser Vergleich verdeutlicht, dass die Signifikanz aus der Differenz zwischen EG 3 (Leichte Sprache + Fotos) und EG 4 (Keine Unterstützungsmaßnahme) mit $p=0,002$ sowie aus der Differenz zwischen EG 1 (Leichte Sprache) und EG 4 (Keine Unterstützungsmaßnahme) mit $p=0,013$ resultiert. Dementsprechend haben die Schülerinnen und Schüler in EG 1 (Leichte Sprache) und EG 3 (Leichte Sprache+Fotos) signifikant weniger Items ausgelassen als die Lernenden in EG 4 (Keine Unterstützungsmaßnahme). Nach Cohen handelt es sich bei dem Unterschied zwischen EG 1 (Leichte Sprache) und EG 4 (Keine Unterstützungsmaßnahme) mit $r=0,25$ um einen kleinen bis mittleren Effekt ${ }^{7}$. Die Differenz zwischen EG 3 (Leichte Sprache+Fotos) und

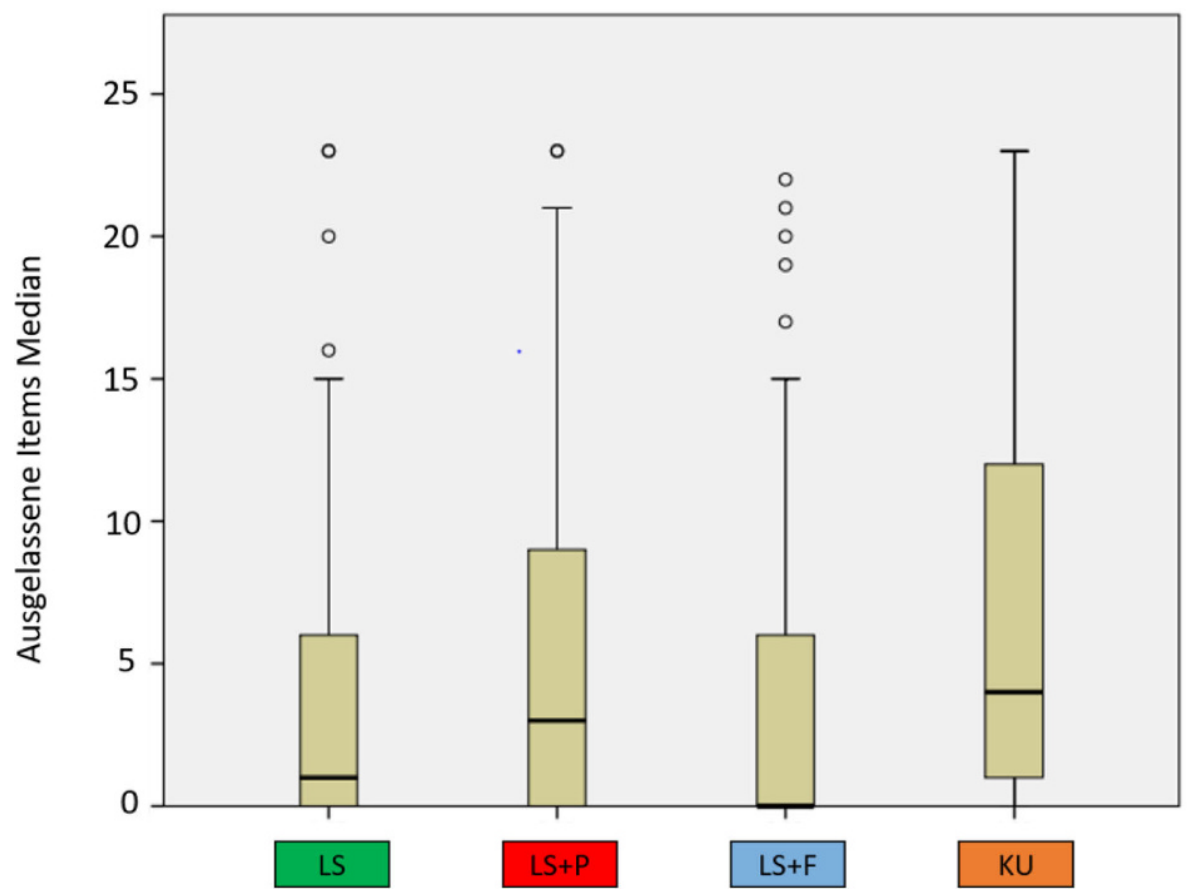

Abb. 12 Boxplot Ausgelassene Items ( $L S$ Leichte Sprache, $L S+P$ Leichte Sprache+ Piktogramme, $L S+F$ Leichte Sprache + Fotos, $K U$ Keine Unterstützungsmaßnahme)

\footnotetext{
7 Zur Angabe der Größe eines non-parametrischen paarweisen Vergleichs wird gemäß Field (2013) das Effektstärkemaß $r$ verwendet.
} 
EG 4 (Keine Unterstützungsmaßnahme) entspricht mit $r=0,29$ einem fast mittleren Effekt (Field 2013) ${ }^{8}$.

Bezüglich der Häufigkeit der falsch bearbeiteten Items konnte kein signifikanter Unterschied zwischen den Experimentalgruppen festgestellt werden $(H(3)=1,96$; $p=0,581$ n. s.). Der Median bezüglich der falsch bearbeiteten Items beträgt für EG 1 (Leichte Sprache) 5, für EG 2 (Leichte Sprache + Piktogramme) 4, für EG 3 (Leichte Sprache+Fotos) 4 und für EG 4 (Keine Unterstützungsmaßnahme) 4 .

\subsection{Extremfälle}

Das übergeordnete Ziel dieses Projektes besteht darin, Erkenntnisse zu gewinnen, wie Lesebarrieren reduziert werden können, sodass möglichst alle Lernenden unabhängig von ihrer Lesefertigkeit auf den mathematischen Inhalt von Aufgaben zugreifen können. Aus diesem Grund ist es, um einen vertieften Einblick in die Wirksamkeit der einzelnen Vereinfachungsüberlegungen zu erhalten, interessant, sich mit den Extremfällen in Bezug auf Lesen auseinanderzusetzen (Bryman 2012) und so der Frage nachzugehen, wie Lernende, die über eine sehr eingeschränkte Lesefertigkeit in der deutschen Sprache verfügen, mit den Aufgaben umgegangen sind. In der vorliegenden Stichprobe erreichte eine Schülerin und ein Schüler im SLS 2-9 keinen Punkt, was bedeutet, dass sie im Lesetest keinen der Sätze durch selbstständiges Lesen inhaltlich erfassen konnten. Beide Lernenden wurden im Rahmen der quantitativen Analyse EG 3 (Leichte Sprache+Fotos) zugewiesen.

Schüler 57 stammt aus Armenien und lebte zum Erhebungszeitpunkt schon seit einigen Jahren in Deutschland. Gemäß seiner Klassenlehrerin und des Eindrucks der Untersuchungsleiterin beherrscht der Junge die deutsche gesprochene Sprache zum Erhebungszeitpunkt auf einem mittleren Niveau. Schüler 57 konnte jedoch noch nicht selbstständig lesen und hatte einen zugewiesenen SFL ${ }^{9}$. Im Intelligenztest CFT 20-R erreichte er 74 Punkte (unterdurchschnittliche Intelligenz; Mittelwert 100; Streuung 15; Prozentrang $4^{10}$ ).

Schülerin 215 lebte zum Erhebungszeitpunkt seit zwei Wochen in Deutschland und stammt aus Syrien. Das Mädchen sprach noch kein Deutsch und hatte keinen $\mathrm{SPF}^{11}$. Sie erreichte im Intelligenztest CFT 20-R einen Wert von 90 Punkten (durchschnittliche Intelligenz; Mittelwert 100; Streuung 15; Prozentrang 24). Obwohl der CFT 20-R im Manual als sprachfrei beschrieben wird (Weiß und Weiß 2006, S. 30), muss berücksichtigt werden, dass die Instruktionen in deutscher Sprach erklärt werden. Da Schülerin 215 zum Erhebungszeitpunkt noch kein Deutsch sprach, könnte für sie ein Nachteil bei der Bearbeitung des CFT 20-R entstanden sein.

\footnotetext{
${ }^{8}$ Ein Effekt bis $r=0,1$ wird als klein bezeichnet, ab $r=0,3$ spricht man von einem mittleren Effekt und ab $r=0,5$ gilt ein Effekt als stark (Rasch et al. 2014).

9 sonderpädagogischer Förderbedarf im Bereich Lernen.

10 Der Prozentrang verdeutlicht, in welcher Beziehung ein Einzelergebnis zu der Vergleichsgruppe zu interpretieren ist. Ein Prozentrang von 10 bedeutet beispielsweise, dass jemand mit seinem Testwert $9 \%$ der Stichprobe übertrifft und selbst von $90 \%$ übertroffen wird.

11 sonderpädagogischen Förderbedarf.
} 
Tab. 4 Summenscore bei der Bearbeitung der Aufgaben

\begin{tabular}{llll}
\hline & Aufgabenkomplex 1 & Aufgabenkomplex 2 & Insgesamt \\
\hline Schüler 57 & & & \\
Anzahl der richtig bearbeiteten Items & 0 (von 4) & 0 (von 23) & 0 (von 27) \\
Anzahl der falsch bearbeiteten Items & 4 & 6 & 10 \\
Anzahl der ausgelassenen Items & 0 & 17 & 17 \\
Schülerin 215 & & & 15 (von 27) \\
Anzahl der richtig bearbeiteten Items & 0 (von 4) & 15 (von 23) & 1 \\
Anzahl der falsch bearbeiteten Items & 0 & 1 & 11 \\
Anzahl der ausgelassenen Items & 4 & 7 & \\
\hline
\end{tabular}

Tab. 4 zeigt, dass Schüler 57 keines der 27 Items mathematisch korrekt bearbeitet hat. Obwohl Schülerin 215 kein Item des ersten Aufgabenkomplexes lösen konnte, kommt sie im Gegensatz zu Schüler 57 bei 15 Items des zweiten Aufgabenkomplexes zu einer mathematisch korrekten Lösung. Tab. 4 zeigt des Weiteren, dass sich die von Schüler 57 erreichten 0 Punkte im Summenscore aus 10 falsch bearbeiteten und 17 ausgelassenen Items ergeben. Schülerin 215 hat nur ein Item falsch bearbeitet und 11 Items ausgelassen.

\section{Diskussion}

Die im vorherigen Kapitel vorgestellten Ergebnisse und ihre Konsequenzen für die unterrichtliche Praxis sollen nun vor dem Hintergrund der Forschungsfragen sowie theoretischer Ansätze diskutiert werden. Zunächst wird ein Bezug zu den Forschungsfragen hergestellt.

Forschungsfrage 1 Verbessert die Verwendung von Leichter Sprache bzw. Leichter Sprache und Piktogrammen oder Fotos die Performanz im Sinne der Lösungsrate bei der Bearbeitung mathematischer Aufgaben, in welchen es um die Einführung der Bruchzahlen geht?

Gemäß Abschn. 5.1 kann Forschungsfrage 1 mit ,ja“ beantwortet werden, wobei der signifikante Unterschied durch die Differenz des Summenscores von EG 3 (Leichte Sprache+Fotos) und EG 4 (Keine Unterstützungsmaßnahme) entsteht. Dementsprechend führt die Unterstützungsmaßnahme Leichte Sprache + Fotos zu einem signifikant höheren Summenscore und damit zu einer signifikant besseren Bearbeitung der Aufgaben im Vergleich zu einer Bearbeitung ohne Unterstützungsmaßnahme. Die Annahme, dass visuelle Unterstützungsmaßnahmen hauptsächlich zum Erfassen von vollständigen Arbeitsaufträgen hilfreich sind, kann im Rahmen der vorliegenden Studie bestätigt werden (vgl. Noll et al. 2018). Die Repräsentation einzelner Worte durch Piktogrammen reicht zum Verständnis von Arbeitsaufträgen offenbar nicht aus.

Der Unterschied zwischen EG 1 (Leichte Sprache) zu EG 4 (Keine Unterstützungsmaßnahme) ist nicht signifikant, weshalb über die Wirkung von Leichter Sprache keine eindeutige Aussage getroffen werden kann. Dieses Ergebnis geht einher 
mit einer Studie von Kohnen et al. (2017), die den Schluss nahelegt, dass Physiktexte in Leichter Sprache nicht zu einem höheren Textverständnis zu führen scheinen. Auf einer deskriptiven Ebene betrachtet, wird trotz des nicht signifikanten Unterschiedes deutlich, dass EG 1 (Leichte Sprache) durchschnittlich den zweithöchsten Summenscore erreicht.

Die Überlegenheit von EG 3 (Leichte Sprache+ Fotos) gegenüber EG 2 (Leichte Sprache + Piktogramme) könnte wie folgt erklärt werden. Für die Schülerinnen und Schüler in EG 3 (Leichte Sprache+Fotos) ist der sprachliche Anspruch gering, wobei inhaltliche und sprachliche Zusammenhänge durch das Vorhandensein der Fotos verdeutlicht werden können. Beispielsweise lautet der erste Arbeitsauftrag von Aufgabe 2.c „Schaue Quadrate D an“. Es folgt die Arbeitsanweisung „Male die blauen Puzzle-Teile in das Quadrat“. Durch die Abfolge der Aufgaben ist klar, dass sich die Arbeitsanweisung „Male die blauen Puzzle-Teile in das Quadrat“ auf die zuvor dargelegte Situation bezieht. Dies wird jedoch durch das Foto (Abb. 13) im Gegensatz zu den Piktogrammen (Abb. 14) deutlich. Auch die geringe kognitive Anregung der Arbeitsaufträge in Leichter Sprache könnte durch die verdeutlichende Wirkung von Fotos ausgeglichen werden.

Ein Vergleich der Mittelwerte von EG 1 (Leichte Sprache) und EG 2 (Leichte Sprache + Piktogramme) zeigt, dass die Piktogramme die Schülerinnen und Schüler im Durchschnitt eher zu irritieren scheinen. Die hier genutzte Verwendung von Piktogrammen stellt vermutlich nicht für alle Kinder eine Vereinfachung dar. Die vorgestellte Strategie setzt auf die Verständnisunterstützung einzelner Wörter eines Satzes durch Piktogramme. Aus der rein piktografischen Darstellung lässt sich so die Bedeutung bzw. der Arbeitsauftrag nicht vollständig unabhängig vom Text erschließen, weshalb die grundlegende Unterstützung des Erfassens des gesamten Arbeitsauftrages durch die Art der Darstellung nicht vorhanden ist. Zudem kann die Fülle an bildlichen Darstellungen überfordern und eine Ablenkung darstellen.

Abb. 13 Aufgabe 2.c (Leichte Sprache + Fotos)

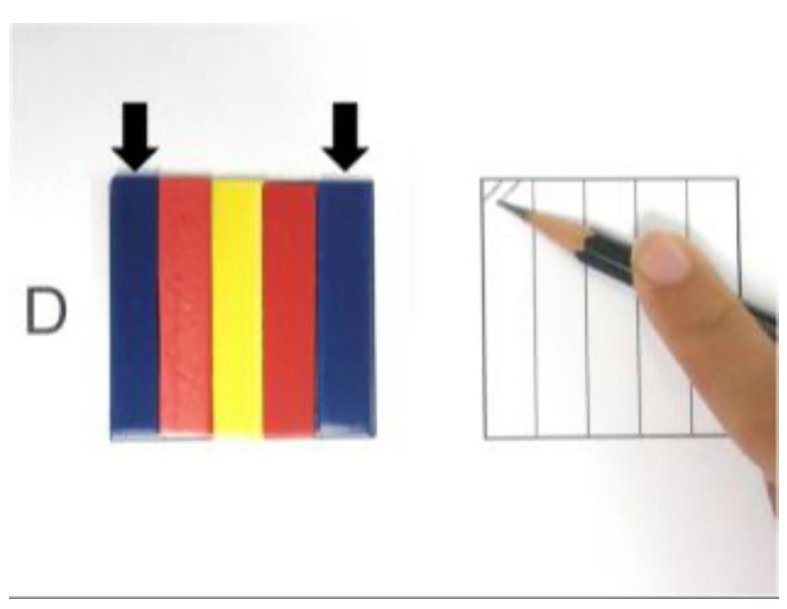



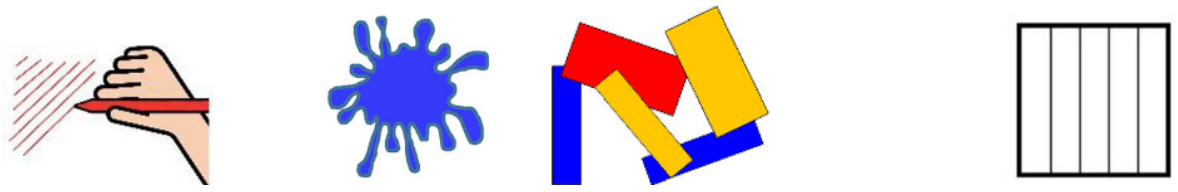

Abb. 14 Aufgabe 2.c (Leichte Sprache + Piktogramme)

Forschungsfrage 2 Unterscheiden sich die Schülerinnen und Schüler in den Experimentalgruppen bezüglich ihrer Performanz, wenn die Lesefertigkeit und der IQ Lernenden berücksichtigt wird?

Wie in Abschn. 5.2 dargestellt, ist die Beziehung zwischen Lesefertigkeit und Summenscore in jeder Experimentalgruppe ähnlich. Mit steigender Lesefertigkeit steigt der Summenscore, wobei die Schülerinnen und Schüler in EG 3 (Leichte Sprache + Fotos) durchschnittlich den höchsten und die Lernenden in EG 4 (Keine Unterstützungsmaßnahme) durchschnittlich den niedrigsten Summenscore erreichen. Bei starken und schwachen Lesern ist der Effekt der Bedingung dementsprechend vergleichbar. Dieses Ergebnis scheint zunächst in Widerspruch zu vorherigen Studien zu stehen, die zeigen, dass Leserinnen und Leser in Abhängigkeit ihrer Lesefähigkeit unterschiedlich von der Kohäsion eines Textes profitieren (McNamara und Kintsch 1996; McNamara et al. 2016). Eine Erklärung für diese kontroversen Befunde könnte darin begründet sein, dass in der vorliegenden Studie im Gegensatz zu den Untersuchungen von McNamara und Kintsch (1996) sowie McNamara et al. (2016) das Erfassen einzelner Sätze, nicht aber das Textverständnis, zentral war.

Die Tatsache, dass der Effekt der Bedingungen bei starken und schwachen Lesern vergleichbar ist, kann auf mehrere Arten interpretiert werden. Zunächst soll sich des Integrated Model of Text and Picture Comprehension (Schnotz 2014) sowie der Cognitive Theory of Mutimedia Learning (Mayer 2009) bedient werden. Gemäß dieser Theorien werden visuelle und sprachliche Informationen mental in unterschiedlichen Kanälen verarbeitet. Durch das Vorhandensein von Fotos haben die Lernenden die Möglichkeit, verbale und visuelle mentale Modelle zu konstruieren und Verbindungen zwischen diesen Modellen aufzubauen, was sich positiv auf den Verstehensprozess auswirken kann (Mayer 2009, S. 23). Hinzu kommt, dass das Vorhandensein der Fotos dazu führen kann, dass weniger kognitive Kapazität zur Enkodierung der Aufgabenstellung benötigt wird. Gemäß der Cognitive Load Theory (Sweller 1988; Ayres et al. 2011) könnte die lernirrelevante kognitive Belastung dadurch reduziert und das Arbeitsgedächtnis entlastet werden, wodurch allen Schülerinnen und Schülern mehr Kapazität bleibt, die für lernrelevante Prozesse eingesetzt werden konnte. Durch diese erhöhte Kapazität bedingt, könnten sowohl starke als auch schwache Leser von der Unterstützungsmaßnahme Leichte Sprache + Fotos profitieren. Die Schlussfolgerung, dass die Verarbeitung von Fotos eine geringere kognitive Kapazität erfordert als beispielsweise die Verarbeitung von Piktogrammen, geht einher mit früheren Studien (Mirenda und Locke 1989; Sevcik und Romski 1986).

Eine weitere Interpretation dieses Ergebnisses könnte sein, dass Fotos zusätzliche Information (z. B. Visualisierung des Ergebnisses einer vorausgegangenen Aufgabe; methodische Hilfestellung, da das Foto zeigt, wie die Aufgabe zu bearbeiten ist) 
liefern, wovon sowohl starke als auch schwache Leser profitieren. Diese Zusatzinformation könnte ein Grund für die generelle Überlegenheit der Lernenden in EG 3 (Leichte Sprache+Fotos) darstellen. Nun könnte man argumentieren, dass zusätzliche Informationen zu einem unverhältnismäßig größeren Vorteil für EG 3 (Leichte Sprache+Fotos) führen. Dass Fotos einen Hinweis geben, ist jedoch ein charakteristischer Vorteil dieses Mediums. Es liegt in der Natur multimedialer Repräsentationen, dass sie nicht nur redundante, sondern auch zusätzliche Informationen bieten (Mayer 2009). Da das Ziel dieser Studie darin besteht, Ergebnisse mit einer hohen Relevanz für den Unterricht zu generieren, ist dieser Unterschied und der damit verbundene Vorteil der Fotos als wichtiger anzusehen als eine höhere Vergleichbarkeit der Settings. Ein Vergleich verschiedener Unterstützungsmaßnahmen, der charakteristische Vorteile ausklammert, hätte nur eine geringe Aussagekraft für deren Einsatz im Unterricht. Des Weiteren soll an dieser Stelle angemerkt werden, dass, obwohl im Rahmen der Fotos Hilfestellungen gegeben werden, die Lösung der Aufgabe in keinem Foto visualisiert wird.

Abschließend soll darauf hingewiesen, dass auch die Beziehung zwischen IQ und Summenscore in jeder Experimentalgruppe ähnlich ist. Mit steigendem IQ steigt der Summenscore, wobei die Schülerinnen und Schüler in EG 3 (Leichte Sprache + Fotos) durchschnittlich den höchsten und die Lernenden in EG 4 (Keine Unterstützungsmaßnahme) durchschnittlich den niedrigsten Summenscore erreichen. Bei Schülerinnen und Schülern mit hohem und niedrigem IQ ist der Effekt der Bedingung dementsprechend vergleichbar. Dieses Ergebnis geht einher mit einer Untersuchung von Langer et al. (2011, S. 200, S. 215).

Forschungsfrage 3 Führen die Unterstützungsmaßnahmen zu mehr Lösungsversuchen?

Gemäß den in Abschn. 5.3 dargestellten Ergebnissen haben die Schülerinnen und Schüler in EG 1 (Leichte Sprache) und EG 3 (Leichte Sprache+Fotos) signifikant weniger Aufgaben ausgelassen als die Lernenden in EG 4 (Keine Unterstützungsmaßnahme). Die Anzahl der ausgelassenen Aufgaben von EG 2 (Leichte Sprache + Piktogramme) unterscheidet sich nicht signifikant von EG 4 (Keine Unterstützungsmaßnahme). Bei der Differenz zwischen EG 1 (Leichte Sprache) und EG 4 (Keine Unterstützungsmaßnahme) handelt es sich um einen kleinen bis mittleren, beim Unterschied zwischen EG 3 (Leichte Sprache + Fotos) und EG 4 (Keine Unterstützungsmaßnahme) um einen mittleren Effekt. Die Anzahl der ausgelassenen Items repräsentiert, wie gut die Schülerinnen und Schüler verstehen, was bei der jeweiligen Aufgabe zu tun ist, unabhängig davon, ob die Aufgabe richtig oder falsch bearbeitet wurde. Dementsprechend bildet die Anzahl der ausgelassenen Items die Merkmalsausprägung „Anzahl der Lösungsversuche“ ab und Forschungsfrage 3 kann für die Lernenden in EG 1 (Leichte Sprache) und EG 3 (Leichte Sprache+Fotos) bejaht werden. Durch diese gesteigerte Anzahl an Lösungsversuchen eröffneten sich signifikant mehr Lerngelegenheiten. Unabhängig davon, ob die Schülerinnen und Schüler eine Aufgabe mathematisch korrekt bearbeiteten, konnte ihre Hemmung, eine Aufgabe überhaupt zu bearbeiten, durch Leichte Sprache und Fotos reduziert werden. Die Nichtbeachtung der mathematischen Korrektheit der Lösung weist dementsprechend in eine sehr ähnliche Richtung wie der Vergleich der Experimentalgruppen auf 
Basis einer richtig-falsch-Kodierung. Lässt man die mathematische Korrektheit der Lösung außen vor, wird jedoch ein deutlicherer Unterschied zwischen EG 1 (Leichte Sprache) und EG 4 (Keine Unterstützungsmaßnahmen) sichtbar. Die Häufigkeit der falsch bearbeiteten Items unterscheidet im Durchschnitt nur minimal zwischen den Experimentalgruppen. Dies war aufgrund der kurzen Bearbeitungszeit nicht anders zu erwarten.

Nachdem die Forschungsfragen diskutiert wurden, soll nun auf die beiden Extremfälle eingegangen werden (vgl.Abschn. 5.4). Die Ergebnisse lassen vermuten, dass sowohl Schülerinnen und Schüler mit schlechten bis sehr schlechten Deutschkenntnissen als auch Schülerinnen und Schüler mit sehr eingeschränkter Lesefertigkeit von der Unterstützungsmaßnahme Leichte Sprache + Fotos profitieren könnten. Warum die Ergebnisse der beiden Lernenden so unterschiedlich ausfallen, kann auf Basis dieser Daten nur gemutmaßt werden. Weitere Studien, die auf Basis einer repräsentativen Stichprobe untersuchen, wie eingeschränkte Deutsch- oder Lesekenntnisse die Nutzung der Unterstützungsmaßnahme beeinflussen, sind notwendig, um diese Frage beantworten zu können.

Aus den in Abschn. 5 dargestellten und hier diskutierten Ergebnissen lassen sich Implikationen für die schulische Praxis ableiten. Diese werden im Folgenden vorgestellt und kritisch beleuchtet. Zunächst kann die Verwendung von Fotos in Kombination mit Leichter Sprache zur Erleichterung des Erfassens von Aufgabenstellungen empfohlen werden. Dabei muss berücksichtigt werden, dass die Gestaltung der Fotos keinesfalls beliebig sein darf (vgl. Abschn. 4.2). Ein Vergleich der Mittelwerte von EG 3 (Leichte Sprache + Fotos) und EG 4 (Keine Unterstützungsmaßnahme) zeigt, dass es sich nach Cohen lediglich um einen kleinen bis mittleren Effekt handelt $(d=0,448)$. Unter Berücksichtigung der kurzen Bearbeitungszeit von durchschnittlich ca. 21 min kann jedoch auch ein Effekt dieser Größenordnung für die Gestaltung von Arbeitsaufträgen in der Praxis sehr bedeutsam sein.

Neben der mathematisch korrekten Lösung wurde auch die Anzahl der ausgelassenen Aufgaben zwischen den Experimentalgruppen verglichen. Dieser Vergleich der bearbeiteten Aufgaben unabhängig von der Korrektheit der Lösung zeigte eine deutliche Überlegenheit von EG 3 (Leichte Sprache+Fotos) und EG 1 (Leichte Sprache) gegenüber EG 4 (Keine Unterstützungsmaßnahme). Für die schulische Praxis bedeutet dies, dass vor allem die Kombination von Fotos und Leichter Sprache, aber auch die alleinige Verwendung von Leichter Sprache den Lernenden signifikant mehr Lerngelegenheiten eröffnet.

Während der Einsatz von Leichter Sprache in Kombination mit Fotos gewinnbringend zu sein scheint, kann der Einsatz von Leichter Sprache und Piktogrammen an dieser Stelle nicht empfohlen werden. Vor allem unter Berücksichtigung des mit dem Einsatz von Piktogrammen verbundenen Mehraufwandes für die Lehrperson scheint die Verwendung von Piktogrammen für die untersuchte Schülerschaft nicht sinnvoll. Obwohl sich die praktische Umsetzung aller Unterstützungsmaßnahmen teilweise als schwierig erwies, kann die adäquate Verwendung von Piktogrammen im Nachhinein als am anspruchsvollsten und zeitaufwendigsten beschrieben werden. Dies liegt daran, dass für einige Schlüsselwörter kein passendes transparentes oder transluzentes Piktogramm in der verwendeten Symbolsammlung Metacom (Kitzinger 2015) zur Verfügung steht. Teilweise wurden Piktogramme in der vorliegenden 
Studie selbst hergestellt, wie z.B. das Piktogramm für ,größer“. Dieser Prozess ist jedoch sehr zeitaufwendig und im Schulalltag sicherlich nicht zu bewerkstelligen. Dementsprechend muss in diesen Fällen individuell entschieden werden, ob das Schlüsselwort mit einem abstrakten oder keinem Piktogramm verknüpft wird (Noll et al. 2020). Im Gegensatz zur Selbstentwicklung von Piktogrammen ist die Aufnahme von Fotos weniger zeitaufwendig. Obwohl in der vorliegenden Studie jedes Fotos selbst aufgenommen wurde, kann die Herstellung der Unterstützungsmaßnahme „Leichte Sprache + Fotos“ als wesentlich weniger zeitaufwendig beschrieben werden als die Auswahl vorgefertigter Piktogramme.

Da die vier Experimentalgruppen im Durchschnitt ein sehr ähnliches Zeitfenster für die Bearbeitung der Aufgaben benötigten, wäre es für die unterrichtliche Praxis aus organisatorischer Sicht unproblematisch, die Schülerinnen und Schüler mit verschiedenen Unterstützungsmaßnahme arbeiten zu lassen. Wie bereits erwähnt, profitieren jedoch sowohl starke als auch schwache Leserinnen und Leser von der Unterstützungsmaßnahme „Leichte Sprache + Fotos“ gleichermaßen. Gleiches gilt sowohl für Schülerinnen und Schüler mit hoher als auch mit niedriger kognitiver Fähigkeit. Demensprechend kann auf Basis der vorliegenden Studie keine Differenzierung bei der Verwendung der Unterstützungsmaßnahme Leichte Sprache + Fotos empfohlen werden. Hinzu kommt, dass die Analyse der beiden Extremfälle die Schlussfolgerung nahelegt, dass sowohl Lernende mit schlechten bis sehr schlechten Deutschkenntnissen als auch Lernende mit sehr eingeschränkter Lesefertigkeit von der Unterstützungsmaßnahme Leichte Sprache + Fotos profitieren könnten.

Dies soll natürlich nicht implizieren, dass das Lernmaterial aller Schülerinnen und Schüler zu jeder Zeit mit Hilfe von Leichter Sprache und Fotos vereinfacht werden sollte. Die Unterstützungsmaßnahmen sollten gezielt bei komplexen, neuen Themengebieten eingesetzt werden, um möglichst allen Lernenden die Bearbeitung der Aufgaben zu ermöglichen. Die Unterstützungsmaßnahme Fotos + Leichte Sprache sollte jedoch nicht als universelle Lösung angesehen werden. Die gezielte Förderung von Lesefertigkeiten darf keinesfalls im Unterricht vernachlässigt werden. Wenn zu jeder Zeit eine sprachliche und visuelle Vereinfachung von Aufgabenstellungen stattfinden würde, würde dies einer adäquaten Lebensvorbereitung der Schülerinnen und Schüler nicht gerecht werden. Den Unterstützungsmaßnahmen könnte dadurch statt einer inklusiven eine exkludierende Wirkung zukommen (Zurstrassen 2015, S. 130). Eine Förderung der Lernenden bei der Enkodierung von nicht vereinfachter, in Schriftsprache dargebotener Informationen ist wichtig. Andernfalls würden sie von vielen gesellschaftlichen Bereichen ausgeschlossen werden. Dennoch sind derartige Vereinfachungen aufgrund der Ausgangsbedingungen einzelner Schülerinnen und Schüler essentiell, um überhaupt auf komplexe Inhalte zugreifen zu können (Scholz et al. 2018), da sie nicht, noch nicht oder vielleicht auch nicht in absehbarer Zeit über entsprechende Fähigkeiten verfügen.

Abschließen soll auf einige Limitationen eingegangen werden. Es muss zunächst darauf hingewiesen werden, dass die dargestellten Ausführungen nur für die vorliegenden Aufgaben gelten, in welchen es um ein spezielles Themengebiet (elementares Bruchzahlenwissen) geht. Des Weiteren arbeiteten die Schülerinnen und Schüler in Einzelarbeit. Dementsprechend können daraus keine Schlussfolgerungen zu kooperativen Arbeitsformen gezogen werden. Dies schränkt, ebenso wie die Tat- 
sache, dass die Rolle der Lehrkraft in der Studie unbeachtet bleibt, die ökologische Validität der Studie ein. Bedacht werden sollte auch, dass für das Aufbauen von Verständnis durch die Bearbeitung von Aufgaben die Ermöglichung der Erfassung der Aufgaben eine unabdingbare Voraussetzung ist. Dies bedeutet jedoch nicht, dass sich inhaltliches Verständnis entwickelt. Hierzu sind weitere Voraussetzungen (z. B. Motivation) ausschlaggebend. Außerdem sollte berücksichtigt werden, dass die angenommene Wirksamkeit von Leichter Sprache auf Basis der Studie nicht bestätigt werden kann. Lediglich die Kombination aus Leichter Sprache und Fotos hatte einen signifikanten Effekt auf die mathematisch richtige Aufgabenbearbeitung. Inwieweit die sprachlichen Anpassungen in Kombination mit den Fotos ausschlaggebend waren oder ob nur die Fotos, welche nicht die sprachliche Ebene beeinflussen, den Effekt verursachten, kann auf Basis der Studie nicht näher analysiert werden. Des Weiteren stellt sich die Frage, inwiefern die ausgewählten Unterstützungsmaßnahmen überhaupt vergleichbar sind, bzw. ob dieser Vergleich fair ist. Beispielsweise muss kritisch angemerkt werden, dass die Lernenden in EG 3 (Leichte Sprache+ Fotos) im Rahmen der Fotos eine Zusatzinformation erhalten. Dies kann zu ihrer generellen Überlegenheit bei der Bearbeitung der Aufgaben geführt haben. Gleiches gilt für den Einsatz Leichter Sprache. Obwohl in Leichter Sprache formulierte Arbeitsaufträge oft weniger ausführlich sind, liegt ihr Vorteil in der daraus resultierenden geringeren sprachlichen Komplexität. Im Unterricht würde die Unterstützungsmaßnahme Leichte Sprache + Fotos gerade wegen ihrer Vorteile zum Einsatz kommen, weshalb die Vorteile der Unterstützungsmaßnahme im Rahmen der Studie nicht ausgeblendet wurden.

Zusammenfassend sei festgehalten, dass aus Gründen der sprachlichen Förderung der Schülerinnen und Schüler und aufgrund des zeitlichen Aufwands für die Lehrkraft der Einsatz der Unterstützungsmaßnahme Leichte Sprache + Fotos nicht als universelle Lösung, sondern zur gezielten Verwendung bei komplexen Themengebieten empfohlen wird. Die Unterstützungsmaßnahme Leichte Sprache + Fotos könnte neben einer gezielten Verwendung im Unterricht auch an außerschulischen Lernorten eingesetzt werden. Beispielsweise ist das selbstständige Erfassen von Arbeitsaufträgen für die Bearbeitung mathematischer Aufgaben in Schülerlaboren zentral (vgl. Scholz et al. 2016b). Darüber hinaus könnte die Unterstützungsmaßnahme im Sinne eines Nachteilsausgleich bei Schulleistungsstudien zum Einsatz kommen, indem Arbeitsaufträge mit Hilfe von Fotos und Leichter Sprache in vereinfachter Form dargestellt werden. Eine andere Transferstrategie stellt die Zusammenarbeit mit Schulbuchverlagen dar.

Ein nächster Schritt könnte darin bestehen die Perspektive von Lehrkräften aufzugreifen. Es sollte untersucht werden, inwiefern Lehrkräfte auf Basis der vorliegenden Ergebnisse dazu bereit wären, Lernmaterialien durch Unterstützungsmaßnahmen zu vereinfachen. Eine Studie von Kambouri et al. (2016) gibt Grund zu der Annahme, dass Lehrkräfte der Verwendung von Piktogrammen positiv gegenüberstehen, jedoch nur unzureichend über diese mögliche Unterstützungsmaßnahme informiert sind. Daher kann die Entwicklung eines Fortbildungsprogramms für den praktischen Einsatz der Unterstützungsmaßnahmen im Unterricht empfohlen werden. In einem solchen Fortbildungsprogramm könnten beispielsweise Gestaltungskriterien thematisiert werden (vgl. Abschn. 4.2). Darüber hinaus sollte in sich anschließen- 
de Forschungsvorhaben eine Erweiterung der Stichprobe (z.B. um andere Förderschwerpunkte) sowie eine Variation des Themengebiets und der Textsorte angestrebt werden.

Funding Open Access funding provided by Projekt DEAL.

Open Access Dieser Artikel wird unter der Creative Commons Namensnennung 4.0 International Lizenz veröffentlicht, welche die Nutzung, Vervielfältigung, Bearbeitung, Verbreitung und Wiedergabe in jeglichem Medium und Format erlaubt, sofern Sie den/die ursprünglichen Autor(en) und die Quelle ordnungsgemäß nennen, einen Link zur Creative Commons Lizenz beifügen und angeben, ob Änderungen vorgenommen wurden.

Die in diesem Artikel enthaltenen Bilder und sonstiges Drittmaterial unterliegen ebenfalls der genannten Creative Commons Lizenz, sofern sich aus der Abbildungslegende nichts anderes ergibt. Sofern das betreffende Material nicht unter der genannten Creative Commons Lizenz steht und die betreffende Handlung nicht nach gesetzlichen Vorschriften erlaubt ist, ist für die oben aufgeführten Weiterverwendungen des Materials die Einwilligung des jeweiligen Rechteinhabers einzuholen.

Weitere Details zur Lizenz entnehmen Sie bitte der Lizenzinformation auf http://creativecommons.org/ licenses/by/4.0/deed.de.

\section{Literatur}

\section{Verwendete Literatur}

Abbott, C. (2000). Symbols now. Hampshire: Ashford Colour.

Ayres, P., Kalyuga, S., \& Sweller, J. (2011). Cognitive load theory. New York: Springer.

Ballod, M. (2001). Verständliche Wissenschaft. Ein informationsdidaktischer Beitrag zur Verständlichkeitsforschung. Tübingen: Gunter Narr.

Ballstaedt, S.-P. (1997). Wissensvermittlung. Die Gestaltung von Lernmaterial. Weinheim: Beltz.

Ballstaedt, S.-P. (2012). Visualisieren. Bilder in wissenschaftlichen Texten. Konstanz: UVK.

Bamberger, R., \& Vanecek, E. (1984). Lesen-Verstehen-Lernen-Schreiben. Die Schwierigkeitsstufen von Texten in deutscher Sprache. Wien: Verlag Jugend und Volk.

Basendowski, S. (2014). Grundbildung - Ein Konzept für alle in einem inklusiven Bildungssystem? Vierteljahreszeitschrift für Heilpädagogik und ihre Nachbargebiete, 3, 191-204.

Best, K. (2006). Sind Wort- und Satzlänge brauchbare Kriterien zur Bestimmung der Lesbarkeit von Texten? In S. Wichter \& A. Busch (Hrsg.), Wissenstransfer Erfolgskontrolle und Rückmeldungen aus der Praxis (S. 21-32). Frankfurt a.M.: Peter Lang.

Bryman, A. (2012). Social research methods (4. Aufl.). Oxford: Oxford University Press.

Bühner, M. (2006). Einführung in die Test- und Fragebogenkonstruktion (2. Aufl.). München: Pearson.

Bundesministerium für Arbeit und Soziales (2014). Leichte Sprache. Ein Ratgeber. https://www. bmas.de/SharedDocs/Downloads/DE/PDF-Publikationen/a752-ratgeber-leichte-sprache.\%20pdf; jsessionid=496CB532ADF5B2274A8E8BC9CBA63C30?_blob=publicationFile\&v\%20=\%204. Zugegriffen: 30. Nov. 2018.

Cardone, D. (1999). Exploring the use of question methods: pictures do not always help people with learning disabilities. The British Journal of Development Disabilities, 45(2), 93-98.

Christmann, U., \& Groeben, N. (1999). Psychologie des Lesens. In B. Franzmann, K. Hasemann, D. Löffler \& E. Schön (Hrsg.), Handbuch Lesen (S. 145-223). München: Saur.

Codling, M., \& Macdonald, N. (2008). User-friendly information: does it convey what it in-tends? Learning Disbility Practice, 11(1), 12-17.

Dehaene, S. (2012). Lesen. Die größte Erfindung der Menschheit und was dabei in unseren Köpfen passiert. München: btb.

Detheridge, T., \& Detheridge, M. (2002). Literacy through symbols: improving access for children and adults (2. Aufl.). London: David Fulton.

Durkin, K., \& Shire, B. (1991). Language in mathematics education-research and practice. Milton Keynes: Open UP.

Eid, M., Gollwitzer, M., \& Schmitt, M. (2010). Statistik und Forschungsmethoden: Lehrbuch (2. Aufl.). Weinheim: Beltz. 
Fajardo, I., Avila, V., Ferrer, A., Tavares, G., Gomez, M., \& Hernandez, A. (2014). Easy-to-read texts for students with intellectual disability: Linguistic factors affecting comprehension. Journal of Applied Research in Intellectual Disabilities, 27, 212-225.

Field, A. (2013). Discovering statistics using IBM SPSS statistics (4. Aufl.). London: SAGE.

Heimlich, U. (2016). Pädagogik bei Lernschwierigkeiten: sonderpädagogische Förderung im Förderschwerpunkt Lernen. Bd. 2. Bad Heilbrunn: Klinkhardt.

Hurtado, B., Jones, L., \& Burniston, F. (2014). Is easy read information really easier to read? Journal of Intellectual Disability Research, 58(9), 822-829.

Jones, F. W., Long, K., \& Finlay, W. M.L. (2007). Symbols can improve the reading comprehension of adults with learning disabilities. Journal of Intellectual Disability Research, 51(7), 545-550.

Kambouri, M., Pampoulou, E. S., Pieridou, M., \& Allen, M. (2016). Science learning and graphic symbols: an exploration of early years teachers' views and use of graphic symbols when teaching science. Eurasia Journal of Mathematics, Science \& Technolo-gy Education, 12(9), 1-19.

Kellermann, G. (2014). Leichte und Einfache Sprache - Versuch einer Definition. Aus Politik und Zeitgeschichte, 9-11, 7-10.

Kitzinger, A. (2015). METACOM 7. Symbolsystem zur Unterstützten Kommunikation

Klicpera, C., Schabmann, A., \& Gasteiger-Klicpera, B. (2014). Legasthenie. Modelle, Diagnose, Therapie und Förderung (4. Aufl.). München: Reinhardt.

KMK (Kultusministerkonferenz) (2003). Bildungsstandards im Fach Mathematik für den Mittleren Schulabschluss. München: Luchterhand.

KMK (Kultusministerkonferenz) (2004). Bildungsstandards im Fach Mathematik für den Hauptschulabschluss. München: Luchterhand.

Kohnen, N., Härtig, H., Berholt, S., \& Retelsdorf, J. (2017). Leichte Sprache im Physikunterricht. In B. Bock, U. Fix \& D. Lange (Hrsg.), „Leichte Sprache“ im Spiegel theoretischer und angewandter Forschung (S. 337-341). Berlin: Frank \& Timm.

Langer, I., Schulz von Thun, F.S., \& Tausch, R. (2011). Sich verständlich ausdrücken. München: Reinhardt.

Laubenstein, D., Lindmeier, C., Guthöhrlein, K., \& Scheer, D. (2015). Auf dem Weg zur schulischen Inklusion: Empirische Befunde zum gemeinsamen Unterricht in rheinlandpfälzischen Schwerpunktschulen. Perspektiven sonderpädagogischer Forschung. Bad Heilbrunn: Klinkhardt.

Maier, H., \& Schweiger, F. (1999). Mathematik und Sprache. Zum Verstehen und Verwenden von Fachsprache im Unterricht. Wien: Oebv \& hpt.

Mayer, R. (2009). Multimedia learning (2. Aufl.). Cambridge: Cambridge University Press.

MBWJK (Ministerium für Bildung, Wissenschaft, Jugend und Kultur) Rheinland-Pfalz (2007). Rahmenlehrplan Mathematik (Klassenstufe 5-9/10). https://lehrplaene.bildung-rp.de/. Zugegriffen: 5. Juli 2018.

MBWW (Minister für Bildung, Wissenschaft und Weiterbildung) Rheinland-Pfalz (o.J.). Lehrplan Mathematik - Schule für Lernbehinderte (Sonderschule). https://lehrplaene.bildung-rp.de/. Zugegriffen: 5. Juli 2018 .

McNamara, D., \& Kintsch, W. (1996). Learning from texts: Effects of prior knowledge and text coherence. Discourse Processes, 22(3), 247-288.

McNamara, D., Kintsch, E., Songer, N., \& Knitsch, W. (1996). Are good Textes always better? Interaction of text coherence, background knowledge and levels of understanding in learning from text. Cognition and Instruction, 14(1), 1-43.

Merten, K. (1995). Inhaltsanalyse

Meyer, M., \& Tiedemann, K. (2017). Sprache im Fach Mathematik. Mathematik im Fokus. Heidelberg: Spektrum.

Miller, G. A. (1956). The magical number seven, plus or minus two: Some limits on our capacity for processing information. Psychological Review, 63(2), 81-97.

Mirenda, P., \& Locke, P. A. (1989). A comparison of symbol transparency in nonspeaking persons with intellectual disabilities. Journal of Speech and Hearing Disorders, 54(2), 131-140. https://doi.org/ 10.1044/jshd.5402.131.

Netzwerk Leichte Sprache (o.J.). Homepage. https://www.leichte-sprache.org/. Zugegriffen: 17. Dez. 2018.

Noll, A. (2020). Lesebarrieren in einem inklusiven Mathematikunterricht überwinden. Ergebnisse einer qualitativen und einer quantitativen Studie. Landauer Beiträge zur mathematikdidaktischen Forschung. Wiesbaden: Springer Spektrum. 
Noll, A., Roth, J., \& Scholz, M. (2018b). Fostering reading comprehension of learning tasks with pictorial symbols. A qualitative study of subjective views and reading paths of children with and without special needs. International Journal of Special Education, 33(3), 616-629.

Padberg, F., \& Wartha, S. (2017). Didaktik der Bruchrechnung (5. Aufl.). Heidelberg: Spektrum.

Poncelas, A., \& Murphy, G. (2007). Accessible information for people with intellectual disabilities. Do symbols really help? Journal of Applied Research in Intellectual Disabilities, 20(5), 466-474.

Prediger, S., Wilhelm, S., Büchter, A., Gürsoy, E., \& Benholz, C. (2015). Sprachkompetenz und Mathematikleistung - Empirische Untersuchung sprachlich bedingter Hürden in den Zentralen Prüfungen 10. Journal für Mathematik-Didaktik, 36(1), 77-104.

Rasch, B., Friese, M., Hofmann, W., \& Naumann, E. (2010). Quantitative Methoden 2: Einführung in die Statistik für Psychologen und Sozialwissenschaftler (3. Aufl.). Heidelberg, Berlin, New York: Springer.

Rasch, B., Friese, M., Hofmann, W., \& Naumann, E. (2014). Quantitative Methoden 1: Einführung in die Statistik für Psychologen und Sozialwissenschaftler (4. Aufl.). Berlin: Springer.

Renner, G., \& Michley, M. (2015). Intelligenzdiagnostik im Vorschulalter. CHC-theoretisch fundierte Untersuchunsplanung und Cross-battery-assessment. Frühförderung interdisziplinär, 34, 67-83.

Rosebrock, C., \& Nix, D. (2006). Forschungsüberblick: Leseflüssigkeit (Fluency) in der amerikanischen Leseforschung und -didaktik. Didaktik Deutsch, 20, 90-112.

Rüstow, N. (2015). Leichte Sprache - eine neue »Kultur« der Beteiligung. In C. Dönges, W. Hilpert \& B. Zurstrassen (Hrsg.), Didaktik der inklusiven politischen Bildung (S. 115-125). Bonn: bpb.

Scherer, P. (1999). Entdeckendes Lernen im Mathematikunterricht der Schule für Lernbehinderte. Theoretische Grundlagen und evaluierte unterrichtspraktische Erprobung (2. Aufl.). Heidelberg: Edition $\mathrm{S}$.

Schnotz, W. (2014). An integrated model of text and picture comprehension. In R. Mayer (Hrsg.), The Cambridge handbook of multimedia learning (2. Aufl. S. 72-103). Cambridge: Cambridge University Press.

Schnotz, W., \& Dutke, S. (2004). Kognitionspsychologische Grundlagen der Lesekompetenz. Mehrebenenverarbeitung anhand multipler Informationsquellen. In U. Schiefele, C. Artelt, W. Schneider \& P. Stanat (Hrsg.), Struktur, Entwicklung und Förderung von Lesekompetenz.Vertiefende Analysen im Rahmen von PISA 2000 (S. 61-100). Wiesbaden: VS.

Scholz, M., Dechant, C., Dönges, C., \& Risch, B. (2018). Naturwissenschaftliche Inhalte für Schülerinnen und Schüler mit kognitiven Beeinträchtigungen aufbereiten. Entwicklung und Evaluation von Lernmaterialien für den Bereich Umweltbildung mithilfe eines Design-Based Research-Ansatzes. Vierteljahresschrift für Heilpädagogik und ihre Nachbargebiete, 87(4), 318-335. https://doi.org/10. 2378/vhn2018.art36d.

Scholz, M., Dönges, C., Risch, B., \& Roth, J. (2016b). Anpassung von Arbeitsmaterialien für selbstständiges Arbeiten von Schülerinnen und Schülern mit kognitiven Beeinträchtigungen in Schülerlaboren. Zeitschrift für Heilpädagogik, 67(7), 318-328.

Scholz, M., Wagner, M., \& Negwer, M. (2016a). Motorische Fähigkeiten und Kompetenzen im Bereich Kulturtechniken von Schülerinnen und Schülern an Schulen mit dem Förderschwerpunkt körperliche und motorische Entwicklung. Heilpädagogische Forschung, 42(4), 191-201.

Sevcik, R., \& Romski, M.A. (1986). Representational matching skills of persons with severe retardation. Augmentative and Alternative Communication, 2(4), 160-164. https://doi.org/10.1080/ 07434618612331273980.

Siegler, R.S., Fazio, L.K., Bailey, D.H., \& Zhou, X. (2013). Fractions: the new frontier for theories of numerical development. Trends in Cognitive Sciences, 17(1), 13-19.

Sutherland, R. J., \& Isherwood, T. (2016). The evidence for easy-read for people with intellectual disabilities: a systematic literature review. Journal of Policy and Practice in Intellectual Disabilities, 13(4), 297-310.

Sweller, J. (1988). Cognitive Load during problem solving. Effects on learning. Cognitive Science, 12(2), $257-285$.

Ward, L., \& Townsley, R. (2005). 'It's about a dialogue. . ' Working with people with learning difficulties to develop accessible information. British Journal of Learning Disabilities, 33(2), 59-64.

Wartha, S., \& vom Hofe, R. (2005). Probleme bei Anwendungsaufgaben in der Bruchrechnung. Mathematik lehren, 128, 10-16.

Wartha, S., \& Wittmann, G. (2009). Lernschwierigkeiten im Bereich der Bruchrechnung und des Bruchzahlbegriffs. In A. Fritz \& S. Schmidt (Hrsg.), Fördernder Mathematikunterricht in der Sekundarstufe I. Rechenschwierigkeiten erkennen und überwinden. Weinheim, Basel: Beltz.

Weiß, R.H., \& Weiß, B. (2006). CFT 20-R: Grundintelligenztest Skala 2, Revision. Göttingen: Hogrefe. 
Werner, B. (2017a). Inklusive Fachdidaktik? Mathematikdidaktische und sonderpädagogische Überlegungen zur Gestaltung zieldifferenter Bildungsangebote im Sekundarbereich. In U. Kortenkamp \& A. Kuzle (Hrsg.), Beiträge zum Mathematikunterricht 2017 (S. 1021-1024). Münster: WTM.

Werner, B. (2017b). Partizipation und Teilhabe: Eckpfeiler eines inklusiven Mathematikunterrichts. Implikationen aus sonderpädagogischer Perspektive. In U. Kortenkamp \& A. Kuzle (Hrsg.), Beiträge zum Mathematikunterricht 2017 (S. 1045-1048). Münster: WTM.

Wilhelm, N. (2016). Zusammenhänge zwischen Sprachkompetenz und Bearbeitung mathematischer Textaufgaben. Quantitative und qualitative Analysen sprachlicher und konzeptueller Hürden. Heidelberg: Spektrum.

Wimmer, H., \& Mayringer, H. (2014). SLS 2-9: Salzburger Lese-Screening für die Schulstufen 2-9. Bern: Hogrefe.

Zentel, P. (2010). Zur Bedeutung von multiplen Repräsentationen beim Lernen mit Computer und Internet für Menschen mit geistiger Behinderung. http://tobias-lib.uni-tuebingen.de/volltexte/2011/5390/pdf/ Schlussfassung.pdf. Zugegriffen: 24. Aug. 2017.

Zustrassen, B. (2015). Inklusion durch Leichte Sprache? Eine kritische Einschätzung. In C. Dönges, W. Hilpert \& B. Zurstrassen (Hrsg.), Didaktik der inklusiven politischen Bildung (S. 126-138). Bonn: bpb.

\section{Weiterführende Literatur}

Dechant, C., Scholz, M., Dönges, C., Kaltenbach, R., Risch, B., \& Köppen, K. (2018). Lebensgrundlage Boden. Eine Handreichung mit differenzierten Lernmaterialien für den Unterricht in inklusiven Gruppen. https://phbl-opus.phlb.de/frontdoor/index/index/docId/579. Zugegriffen: 30. Nov. 2018.

Noll, A., Sturm, N., Scholz, M., \& Roth, J. (2018a). Mathematik leicht gemacht: Piktogramme und Leichte Sprache für alle Fälle. In O. Weyrauch \& B. Smieja (Hrsg.), Fächerübergreifender Grundschulunterricht. Beiträge aus Theorie und Praxis (S. 79-98). Bern: Peter Lang. 